

\title{
Log canonical thresholds, F-pure thresholds, and nonstandard extensions
}

\author{
Bhargav Bhatt, Daniel J. Hernández, \\ Lance Edward Miller and Mircea Mustață
}

\begin{abstract}
We present a new relation between an invariant of singularities in characteristic zero (the log canonical threshold) and an invariant of singularities defined via the Frobenius morphism in positive characteristic (the $F$-pure threshold). We show that the set of limit points of sequences of the form $\left(c_{p}\right)$, where $c_{p}$ is the $F$-pure threshold of an ideal on an $n$-dimensional smooth variety in characteristic $p$, coincides with the set of $\log$ canonical thresholds of ideals on $n$-dimensional smooth varieties in characteristic zero. We prove this by combining results of Hara and Yoshida with nonstandard constructions.
\end{abstract}

\section{Introduction}

The connection between invariants of singularities in characteristic zero and positive characteristic is a topic that has recently attracted a lot of attention. Typically, the invariants of singularities that arise in birational geometry are defined via divisorial valuations. In characteristic zero, one can use (log) resolutions of singularities to compute such invariants. On the other hand, in commutative algebra in positive characteristic one defines invariants using the action of the Frobenius morphism. It turns out that these invariants have subtle connections, some of them proven, and some still conjectural; see, for example, [Hara and Watanabe 2002; Hara and Yoshida 2003; Mustață et al. 2005]. The typical such connection involves reduction from characteristic zero to positive characteristic. In this note we describe a different, though related connection. We use nonstandard constructions to study limits of invariants in positive characteristic, where the characteristic tends to infinity, in terms of invariants in characteristic zero.

The invariants we study in this paper are the log canonical threshold (in characteristic zero) and the $F$-pure threshold (in positive characteristic). The log canonical threshold is an invariant that plays an important role in birational geometry; see

Hernández was partially supported by RTG grant 0502170 . Mustață was partially supported by NSF grant DMS-0758454 and a Packard Fellowship.

MSC2010: primary 13A35; secondary 13L05, 14B05, 14F18.

Keywords: $F$-pure threshold, log canonical threshold, ultrafilters, multiplier ideals, test ideals. 
[Kollár 1997; Ein and Mustaţă 2006]. Given an irreducible, smooth scheme $X$ defined over a field $k$ of characteristic zero, and a proper ideal $\mathfrak{a} \subset \mathrm{O}_{X}$, the $\log$ canonical threshold of $\mathfrak{a}$ is denoted by $\operatorname{lct}(\mathfrak{a})$. For the precise definition in terms of a $\log$ resolution of $(X, \mathfrak{a})$, we refer to Section 2. Given a point $x \in V(\mathfrak{a})$, one defines $\operatorname{lct}_{x}(\mathfrak{a})$ to be $\operatorname{lct}\left(\left.\mathfrak{a}\right|_{U}\right)$, where $U$ is a small enough open neighborhood of $x$ in $X$.

On the other hand, suppose that $W$ is a smooth scheme of finite type over a perfect field $L$ of positive characteristic $p$. For a proper ideal $\mathfrak{a} \subset O_{W}$, the $F$-pure threshold fpt(a) was introduced and studied in [Takagi and Watanabe 2004]. Given $x \in V(\mathfrak{a})$, one defines as before the local version of this invariant, denoted $\operatorname{fpt}_{x}(\mathfrak{a})$. The original definition of the $F$-pure threshold involved notions and constructions from tight closure theory. However, since we always assume that the ambient scheme is smooth, one can use an alternative description, following [Mustață et al. 2005; Blickle et al. 2008] (see Section 2 below). Part of the interest in the study of the $F$-pure threshold comes from the fact that it shares many of the formal properties of the log canonical threshold.

Before stating our main result, let us recall the fundamental connection between $\log$ canonical thresholds and $F$-pure thresholds via reduction mod $p$. Suppose that $X$ and $\mathfrak{a} \subset \mathrm{O}_{X}$ are defined over $k$, as above. We may choose a subring $A \subset k$, finitely generated over $\mathbb{Z}$, and models $X_{A}$ and $\mathfrak{a}_{A} \subset \mathcal{O}_{X_{A}}$ for $X$ and $\mathfrak{a}$, respectively, defined over $A$. In particular, given any closed point $s \in \operatorname{Spec} A$, we may consider the corresponding reductions $X_{s}$ and $\mathfrak{a}_{s} \subset \mathrm{O}_{X_{s}}$ defined over the finite residue field of $s$ denoted $k(s)$. One of the main results in [Hara and Yoshida 2003] implies the following relation between $\log$ canonical thresholds and $F$-pure thresholds: after possibly replacing $A$ by a localization $A_{a}$ for some nonzero $a \in A$,

i) $\operatorname{lct}(\mathfrak{a}) \geq \operatorname{fpt}\left(\mathfrak{a}_{s}\right)$ for every closed point in $s \in \operatorname{Spec} A$, and

ii) there is a sequence of closed points $s_{m} \in \operatorname{Spec} A$ with $\lim _{m \rightarrow \infty} \operatorname{char}\left(k\left(s_{m}\right)\right)=\infty$ and such that $\lim _{m \rightarrow \infty} \operatorname{fpt}\left(\mathfrak{a}_{s_{m}}\right)=\operatorname{lct}(\mathfrak{a})$.

It is worth pointing out that a fundamental open problem in the field predicts that in this setting there is a dense set of closed points $S \subset \operatorname{Spec} A$ such that $\operatorname{lct}(\mathfrak{a})=\operatorname{fpt}\left(\mathfrak{a}_{s}\right)$ for every $s \in S$.

We now turn to the description of our main result. For every $n \geq 1$, let $\mathscr{L}_{n}$ be the set of all $\operatorname{lct}(\mathfrak{a})$, where the pair $(X, \mathfrak{a})$ is as above, with $\operatorname{dim}(X)=n$. Similarly, given $n$ and a prime $p$, let $\mathscr{F}(p)_{n}$ be the set of all $\operatorname{fpt}(\mathfrak{a})$, where $(W, \mathfrak{a})$ is as above, with $\operatorname{dim}(W)=n$, and $W$ defined over a field of characteristic $p$. The following is our main result.

Theorem 1.1. For every $n \geq 1$, the set of limit points of all sequences $\left(c_{p}\right)$, where $c_{p} \in \mathscr{F}(p)_{n}$ for every prime $p$, coincides with $\mathscr{L}_{n}$.

A key ingredient in the proof of Theorem 1.1 is provided by ultraproduct constructions. Note that if $c \in \mathscr{L}_{n}$ is given as $c=\operatorname{lct}(\mathfrak{a})$, then the above mentioned 
results in [Hara and Yoshida 2003] (more precisely, property ii) above) imply that $c=\lim _{p \rightarrow \infty} c_{p}$, where for $p \gg 0$ prime, $c_{p}$ is the $F$-pure threshold of a suitable reduction $\mathfrak{a}_{s} \subset \mathrm{O}_{X_{s}}$ with $\operatorname{char}(k(s))=p$. Thus the interesting statement in the above theorem is the converse: given pairs $\left(W_{m}, \mathfrak{a}_{m}\right)$ over $L_{m}$ with $\operatorname{dim}\left(W_{m}\right)=n$, $\lim _{m \rightarrow \infty} \operatorname{char}\left(L_{m}\right)=\infty$, and with $\lim _{m \rightarrow \infty} \operatorname{fpt}\left(\mathfrak{a}_{m}\right)=c$, there is a pair $(X, \mathfrak{a})$ in characteristic zero with $\operatorname{dim}(X)=n$ and such that $c=\operatorname{lct}(\mathfrak{a})$.

It is easy to see that we may assume that each $W_{m}=\operatorname{Spec}\left(L_{m}\left[x_{1}, \ldots, x_{n}\right]\right)$ and $c_{m}=\operatorname{fpt}_{0}\left(\mathfrak{a}_{m}\right)$ for some $\mathfrak{a}_{m} \subseteq\left(x_{1}, \ldots, x_{n}\right)$. If we put $\mathfrak{a}_{m}^{(d)}=\mathfrak{a}_{m}+\left(x_{1}, \ldots, x_{n}\right)^{d}$, we have $\left|\operatorname{fpt}\left(\mathfrak{a}_{m}^{(d)}\right)-\operatorname{fpt}\left(\mathfrak{a}_{m}\right)\right| \leq n / d$ for all $m$ and $d$. Ultraproduct constructions give nonstandard extensions of our algebraic structures. In particular, we get a field $k=\left[L_{m}\right]$ of characteristic zero. Since all ideals $\mathfrak{a}_{m}^{(d)}$ are generated in degree less than or equal to $d$, they determine an ideal $\mathfrak{a}^{(d)}$ in $k\left[x_{1}, \ldots, x_{n}\right]$. The key point is to show that for every $\varepsilon>0$, we have $\left|\operatorname{lct}_{0}\left(\mathfrak{a}^{(d)}\right)-\operatorname{fpt}_{0}\left(\mathfrak{a}_{m}\right)\right|<\varepsilon$ for infinitely many $m$. This easily implies that $\lim _{d \rightarrow \infty} \operatorname{lct}_{0}\left(\mathfrak{a}^{(d)}\right)=c$, and since $\mathscr{L}_{n}$ is closed by [de Fernex and Mustață 2009, Theorem 1.3] (incidentally, this is proved in loc. cit. also by nonstandard arguments), we conclude that $c \in \mathscr{L}_{n}$.

As in [Hara and Yoshida 2003], the result relating the log canonical threshold of $\mathfrak{a}^{(d)}$ and the $F$-pure thresholds of $\mathfrak{a}_{m}^{(d)}$ follows from a more general result relating the multiplier ideals of $\mathfrak{a}^{(d)}$ and the test ideals of $\mathfrak{a}_{m}^{(d)}$ (see Theorem 4.1 below). We prove this by following, with some simplifications, the main line of argument in [ibid.] in our nonstandard setting.

The use of ultraproduct techniques in commutative algebra has been pioneered by Schoutens; see [Schoutens 2010] and the list of references therein. This point of view has been particularly effective for passing from positive characteristic to characteristic zero in an approach to tight closure theory and to its applications. Our present work combines ideas of Schoutens [2005] with the nonstandard approach to studying limits of log canonical thresholds and $F$-pure thresholds from [de Fernex and Mustaţă 2009] and [Blickle et al. 2009], respectively.

The paper is structured as follows. In Section 2 we review the definitions of multiplier ideals and test ideals, and recall how the log canonical threshold and the $F$-pure threshold appear as the first jumping numbers in these families of ideals. In Section 3 we review the basic definitions involving ultraproducts. For the benefit of the reader, we also describe in detail how to go from schemes, morphisms, and sheaves over an ultraproduct of fields to sequences of similar objects defined over the corresponding fields. The proof of Theorem 1.1 is given in Section 4 .

\section{Multiplier ideals and test ideals}

In this section we review the basic facts that we will need about multiplier ideals and test ideals. Both these concepts can be defined under mild assumptions on the 
singularities of the ambient space. However, since our main result only deals with smooth varieties, we will restrict to this setting in order to simplify the definitions.

2A. Multiplier ideals and the log canonical threshold. In what follows we recall the definition and some basic properties of multiplier ideals and log canonical thresholds. For details and further properties, we refer the reader to [Lazarsfeld 2004, §9].

Let $k$ be a field of characteristic zero, and $X$ an irreducible and smooth scheme of finite type over $k$. Given a nonzero ideal ${ }^{1} \mathfrak{a}$ on $X$, its multiplier ideals are defined as follows. Let us fix a $\log$ resolution of the pair $(X, \mathfrak{a})$ : this is a projective, birational morphism $\pi: Y \rightarrow X$ with $Y$ smooth and $\mathfrak{a} \cdot \mathscr{O}_{Y}=\mathscr{O}_{Y}(-F)$ for an effective divisor $F$ such that $F+\operatorname{Exc}(\pi)$ is a divisor with simple normal crossings. Here $\operatorname{Exc}(\pi)$ denotes the exceptional divisor of $\pi$. Such resolutions exist by Hironaka's theorem, since we are in characteristic zero. Recall that $K_{Y / X}$ denotes the relative canonical divisor of $\pi$ : this is an effective divisor supported on $\operatorname{Exc}(\pi)$ such that $\mathcal{O}_{Y}\left(K_{Y / X}\right) \simeq \omega_{Y} \otimes f^{*}\left(\omega_{X}^{-1}\right)$. With this notation, the multiplier ideal of $\mathfrak{a}$ of exponent $\lambda \in \mathbb{R}_{\geq 0}$ is defined by

$$
\Phi\left(\mathfrak{a}^{\lambda}\right):=\pi_{*} \mathcal{O}_{Y}\left(K_{Y / X}-\lfloor\lambda F\rfloor\right) .
$$

Here, for a divisor with real coefficients $E=\sum_{i} a_{i} E_{i}$, we write $\lfloor E\rfloor=\sum_{i}\left\lfloor a_{i}\right\rfloor E_{i}$, where $\left\lfloor a_{i}\right\rfloor$ is the largest integer $\leq a_{i}$. It is a basic fact that the definition of multiplier ideals is independent of resolution.

Let us consider some easy consequences of the definition (1). If $\lambda<\mu$, then $\mathscr{F}\left(\mathfrak{a}^{\mu}\right) \subseteq \mathscr{g}\left(\mathfrak{a}^{\lambda}\right)$. Furthermore, given $\lambda$, there is $\varepsilon>0$ such that $\mathscr{F}\left(\mathfrak{a}^{\lambda}\right)=\mathscr{F}\left(\mathfrak{a}^{\mu}\right)$ whenever $\lambda \leq \mu \leq \lambda+\varepsilon$. A positive $\lambda$ is a jumping number of $\mathfrak{a}$ if $\mathscr{f}\left(\mathfrak{a}^{\lambda}\right) \neq \mathscr{F}\left(\mathfrak{a}^{\mu}\right)$ for all $\mu<\lambda$. If we write $F=\sum_{i} a_{i} E_{i}$, it follows from (1) that if $\lambda$ is a jumping number, then $\lambda a_{i} \in \mathbb{Z}$ for some $i$. In particular, we see that the jumping numbers of $\mathfrak{a}$ form a discrete set of rational numbers.

Suppose now that $\mathfrak{a} \neq \mathrm{O}_{X}$. The smallest jumping number of $\mathfrak{a}$ is the $\log$ canonical threshold $\operatorname{lct}(\mathfrak{a})$. Note that if $0 \leq \lambda \ll 1$, then $\mathscr{g}\left(\mathfrak{a}^{\lambda}\right)=\mathcal{O}_{X}$, hence $\operatorname{lct}(\mathfrak{a})=\min \left\{\lambda \mid \mathscr{f}\left(\mathfrak{a}^{\lambda}\right) \neq \mathscr{O}_{X}\right\}$ (this is finite since $\left.\mathfrak{a} \neq \mathscr{O}_{X}\right)$. If $\mathfrak{a} \subseteq \mathfrak{b}$, then $\mathscr{J}\left(\mathfrak{a}^{\lambda}\right) \subseteq \mathscr{g}\left(\mathfrak{b}^{\lambda}\right)$ for all $\lambda$; in particular, we have $\operatorname{lct}(\mathfrak{a}) \leq \operatorname{lct}(\mathfrak{b})$. We make the convention $\operatorname{lct}(0)=0$ and $\operatorname{lct}\left(0_{X}\right)=\infty$.

It is sometimes convenient to also have available a local version of the $\log$ canonical threshold. If $x \in X$, then we put $\operatorname{lct}_{x}(\mathfrak{a}):=\max _{V} \operatorname{lct}\left(\left.\mathfrak{a}\right|_{V}\right)$, where the maximum ranges over all open neighborhoods $V$ of $x$. Equivalently, we have

$$
\operatorname{lct}_{x}(\mathfrak{a})=\min \left\{\lambda \mid \mathscr{g}\left(\mathfrak{a}^{\lambda}\right) \cdot \mathscr{O}_{X, . x} \neq \mathcal{O}_{X, x}\right\}
$$

(with the convention that this is 0 if $\mathfrak{a}=(0)$, and it is infinite if $x \notin V(\mathfrak{a})$ ). Note that given a proper ideal $\mathfrak{a}$ on $X$, there is a closed point $x \in X$ such that $\operatorname{lct}(\mathfrak{a})=\operatorname{lct}_{x}(\mathfrak{a})$.

\footnotetext{
${ }^{1}$ Every ideal sheaf that we consider is assumed to be coherent.
} 
The definition of multiplier ideals commutes with extension of the base field, as follows. For a proof, see the proof of [de Fernex and Mustaţă 2009, Propositions 2.9].

Proposition 2.1. Let $\mathfrak{a}$ be an ideal on $X$. If $k \subset k^{\prime}$ is a field extension, and $\varphi: X^{\prime}=X \times_{\operatorname{Spec} k} \operatorname{Spec} k^{\prime} \rightarrow X$ and $\mathfrak{a}^{\prime}=\mathfrak{a} \cdot O_{X^{\prime}}$, then $\mathscr{F}\left(\mathfrak{a}^{\prime \lambda}\right)=\mathscr{F}\left(\mathfrak{a}^{\lambda}\right) \cdot O_{X^{\prime}}$ for every $\lambda \in \mathbb{R}_{\geq 0}$. In particular, $\operatorname{lct}_{x^{\prime}}\left(\mathfrak{a}^{\prime}\right)=\operatorname{lct}_{\varphi\left(x^{\prime}\right)}(\mathfrak{a})$ for every $x^{\prime} \in X^{\prime}$.

Recall from Section 1 that $\mathscr{L}_{n}$ consists of all nonnegative rational numbers of the form $\operatorname{lct}(\mathfrak{a})$, where $\mathfrak{a}$ is a proper ideal on an $n$-dimensional smooth variety over a field $k$ of characteristic zero. It is clear that equivalently, we may consider the invariants $\operatorname{lct}_{x}(\mathfrak{a})$, where $(X, \mathfrak{a})$ is as above, and $x \in X$ is a closed point. Furthermore, by Proposition 2.1 we may assume that $k$ is algebraically closed. One can show that in this definition we can fix the algebraically closed field $k$ and assume that $X=\mathbb{A}_{k}^{n}$ and obtain the same set; see [ibid., Propositions 3.1 and 3.3]. Furthermore, we will make use of the fact that $\mathscr{L}_{n}$ is a closed set; see [ibid., Theorem 1.3].

2B. Test ideals and the F-pure threshold. In this section we assume that $X$ is an irreducible, Noetherian, regular scheme of characteristic $p>0$. We also assume that $X$ is $F$-finite, that is, the Frobenius morphism $F: X \rightarrow X$ is finite (in fact, most of the time $X$ will be a scheme of finite type over a perfect field, in which case this assumption is clearly satisfied). Recall that for an ideal $J$ on $X$, the $e$-th Frobenius power $J^{\left[p^{e}\right]}$ is generated by $u^{p^{e}}$, where $u$ varies over the (local) generators of $J$.

Suppose that $\mathfrak{b}$ is an ideal on $X$. Given a positive integer $e$, one can show that there is a unique smallest ideal $J$ such that $\mathfrak{b} \subseteq J^{\left[p^{e}\right]}$. This ideal is denoted by $\mathfrak{b}^{\left[1 / p^{e}\right]}$. Given a nonzero ideal $\mathfrak{a}$ and $\lambda \in \mathbb{R}_{\geq 0}$, one has

$$
\left(\mathfrak{a}^{\left[\lambda p^{e}\right\rceil}\right)^{\left[1 / p^{e}\right]} \subseteq\left(\mathfrak{a}^{\left\lceil\lambda p^{e+1}\right\rceil}\right)^{\left[1 / p^{e+1}\right]}
$$

for all $e \geq 1$ (here $\lceil u\rceil$ denotes the smallest integer greater than or equal to $u$ ). By the Noetherian property, it follows that there is an ideal $\tau\left(\mathfrak{a}^{\lambda}\right)$ that is equal to $\left(\mathfrak{a}^{\left\lceil\lambda p^{e}\right\rceil}\right)^{\left[1 / p^{e}\right]}$ for all $e \gg 0$. This is the test ideal of $\mathfrak{a}$ of exponent $\lambda$. For details and basic properties of test ideals, we refer to [Blickle et al. 2008].

It is again clear that if $\lambda<\mu$, then $\tau\left(\mathfrak{a}^{\mu}\right) \subseteq \tau\left(\mathfrak{a}^{\lambda}\right)$. It takes a little argument to show that given any $\lambda$, there is $\varepsilon>0$ such that $\tau\left(\mathfrak{a}^{\lambda}\right)=\tau\left(\mathfrak{a}^{\mu}\right)$ whenever $\lambda \leq \mu \leq \lambda+\varepsilon$; see [ibid., Proposition 2.14]. We say that $\lambda>0$ is an $F$-jumping number of $\mathfrak{a}$ if $\tau\left(\mathfrak{a}^{\lambda}\right) \neq \tau\left(\mathfrak{a}^{\mu}\right)$ for every $\mu<\lambda$. It is proved in [ibid., Theorem 3.1] that if $X$ is a scheme of finite type over an $F$-finite field, then the $F$-jumping numbers of $\mathfrak{a}$ form a discrete set of rational numbers.

The smallest $F$-jumping number of $\mathfrak{a}$ is the $F$-pure threshold fpt $(\mathfrak{a})$. Since $\tau\left(\mathfrak{a}^{\lambda}\right)=\mathfrak{O}_{X}$ for $0 \leq \lambda \ll 1$, the $F$-pure threshold is characterized by

$$
\operatorname{fpt}(\mathfrak{a})=\min \left\{\lambda \mid \tau\left(\mathfrak{a}^{\lambda}\right) \neq \mathfrak{O}_{X}\right\} .
$$


Note that this is finite if and only if $\mathfrak{a} \neq \mathfrak{O}_{X}$. We make the convention that $\operatorname{fpt}(\mathfrak{a})=0$ if $\mathfrak{a}=(0)$.

We have a local version of the $F$-pure threshold: given $x \in X$, we put $\operatorname{fpt}_{x}(\mathfrak{a}):=$ $\max _{V} \operatorname{fpt}\left(\left.\mathfrak{a}\right|_{V}\right)$, where the maximum is over all open neighborhoods $V$ of $x$. It can be also described by

$$
\operatorname{fpt}_{x}(\mathfrak{a})=\min \left\{\lambda \mid \tau\left(\mathfrak{a}^{\lambda}\right) \cdot \mathrm{O}_{X, x} \neq \mathrm{O}_{X, x}\right\},
$$

and it is finite if and only if $x \in V(\mathfrak{a})$. Note that given any $\mathfrak{a}$, there is $x \in X$ such that $\operatorname{fpt}(\mathfrak{a})=\mathrm{fpt}_{x}(\mathfrak{a})$.

We will make use of the following two properties of $F$-pure thresholds.

Proposition 2.2 [Blickle et al. 2008, Proposition 2.13]. If $\mathfrak{a}$ is an ideal on $X$ and $S=\widehat{O_{X, x}}$ is the completion of the local ring of $X$ at a point $x \in X$, then $\tau\left(\mathfrak{a}^{\lambda}\right) \cdot S=\tau\left((\mathfrak{a} \cdot S)^{\lambda}\right)$ for every $\lambda \geq 0$. In particular, $\operatorname{fpt}_{x}(\mathfrak{a})=\operatorname{fpt}(\mathfrak{a} \cdot S)$.

Proposition 2.3 [Blickle et al. 2009, Corollary 3.4]. If $\mathfrak{a}$ and $\mathfrak{b}$ are ideals on $X$, and $x \in V(\mathfrak{a}) \cap V(\mathfrak{b})$ is such that $\mathfrak{a} \cdot \mathscr{O}_{X, x}+\mathfrak{m}^{r}=\mathfrak{b} \cdot \mathscr{O}_{X, x}+\mathfrak{m}^{r}$ for some $r \geq 1$, where $\mathfrak{m}$ is the maximal ideal in $\mathrm{O}_{X, x}$, then

$$
\left|\mathrm{fpt}_{x}(\mathfrak{a})-\mathrm{fpt}_{x}(\mathfrak{b})\right| \leq \frac{\operatorname{dim}\left(\mathscr{O}_{X, x}\right)}{r} .
$$

The local $F$-pure threshold admits the following alternative description, following [Mustaţă et al. 2005]. If $\mathfrak{a}$ is an ideal on $X$ and $x \in V(\mathfrak{a})$, let $v(e)$ denote the largest $r$ such that $\mathfrak{a}^{r} \cdot \mathscr{O}_{X, x} \nsubseteq \mathfrak{m}^{\left[p^{e}\right]}$, where $\mathfrak{m}$ is the maximal ideal in $\mathscr{O}_{X, x}$ (we make the convention $v(e)=0$ if $\mathfrak{a}=0)$. One can show that

$$
\operatorname{fpt}_{x}(\mathfrak{a})=\lim _{e \rightarrow \infty} \frac{v(e)}{p^{e}}
$$

(see [Blickle et al. 2008, Proposition 2.29]). This immediately implies the assertion in the following proposition.

Proposition 2.4. Let $L \subset L^{\prime}$ be a field extension of $F$-finite fields of positive characteristic. If $\mathfrak{a} \subseteq L\left[x_{1}, \ldots, x_{n}\right]$ is an ideal vanishing at the origin, and $\mathfrak{a}^{\prime}=$ $\mathfrak{a} \cdot L^{\prime}\left[x_{1}, \ldots, x_{n}\right]$, then $\mathrm{fpt}_{0}(\mathfrak{a})=\mathrm{fpt}_{0}\left(\mathfrak{a}^{\prime}\right)$.

Recall that we have introduced in Section 1 the set $\mathscr{F}(p)_{n}$ consisting of all invariants of the form $\operatorname{fpt}(\mathfrak{a})$, where $\mathfrak{a}$ is a proper ideal on an irreducible, $n$-dimensional smooth scheme of finite type over $L$, with $L$ a perfect field of characteristic $p$. We can define two other related subsets of $\mathbb{R}_{\geq 0}$. Let $\mathscr{F}(p)_{n}^{\prime}$ be the set of invariants $\mathrm{fpt}_{0}(\mathfrak{a})$, where $\mathfrak{a} \subset L\left[x_{1}, \ldots, x_{n}\right]$ is an ideal vanishing at the origin, and $L$ is an algebraically closed field of characteristic $p$. We also put $\mathscr{F}(p)_{n}^{\prime \prime}$ for the set of all $\operatorname{fpt}(\mathfrak{a})$, where $\mathfrak{a}$ is a proper ideal on an irreducible, regular, $n$-dimensional $F$-finite scheme of characteristic $p$. We clearly have the following inclusions:

$$
\mathscr{F}(p)_{n}^{\prime} \subseteq \mathscr{F}(p)_{n} \subseteq \mathscr{F}(p)_{n}^{\prime \prime} .
$$


Proposition 2.5. $\mathscr{F}(p)_{n}^{\prime}$ is dense in $\mathscr{F}(p)_{n}^{\prime \prime}$ (hence also in $\left.\mathscr{F}(p)_{n}\right)$.

This implies that in Theorem 1.1 we may replace the sets $\mathscr{F}(p)_{n}$ by $\mathscr{F}(p)_{n}^{\prime}$ or by $\mathscr{F}(p)_{n}^{\prime \prime}$.

Proof of Proposition 2.5. Suppose that $\mathfrak{a}$ is a proper ideal on $X$, where $X$ is irreducible, regular, $F$-finite, $n$-dimensional, and of characteristic $p$. Let $c=\operatorname{fpt}(\mathfrak{a})$. We can find $x \in X$ such that $c=\mathrm{fpt}_{x}(\mathfrak{a})$. By Proposition 2.2, we have

$$
c=\operatorname{fpt}\left(\mathfrak{a} \cdot \widehat{\sigma_{X, x}}\right) \text {. }
$$

Note that by Cohen's theorem, we have an isomorphism $\widehat{O_{X, x}} \simeq L \llbracket x_{1}, \ldots, x_{d} \rrbracket$, with $L$ an $F$-finite field, and $d \leq n$. If $\mathfrak{m}$ is the maximal ideal in $\widehat{O_{X, x}}$ and $c_{i}=$ $\operatorname{fpt}\left(\mathfrak{a} \cdot \widehat{O_{X, x}}+\mathfrak{m}^{i}\right)$, then Proposition 2.3 gives $c=\lim _{i \rightarrow \infty} c_{i}$. On the other hand, there are ideals $\mathfrak{b}_{i} \subset L\left[x_{1}, \ldots, x_{d}\right]$ such that $\mathfrak{b}_{i} \cdot \widehat{O_{X, x}}=\mathfrak{a} \cdot \widehat{O_{X, x}}+\mathfrak{m}^{i}$, and another application of Proposition 2.2 gives $c_{i}=\mathrm{fpt}_{0}\left(\mathfrak{b}_{i}\right)$. It is easy to see (for example, from formula (2)) that $c_{i}=\mathrm{fpt}_{0}\left(\mathfrak{b}_{i} \cdot L\left[x_{1}, \ldots, x_{n}\right]\right)$. It now follows from Proposition 2.4 that $c_{i}=\mathrm{fpt}_{0}\left(\mathfrak{b}_{i} \cdot \bar{L}\left[x_{1}, \ldots, x_{n}\right]\right)$, where $\bar{L}$ is an algebraic closure of $L$. Therefore all $c_{i}$ lie in $\mathscr{F}(p)_{n}^{\prime}$, which proves the proposition.

In Section 4 we will use a slightly different description of the test ideals that we now present. More precisely, we give a different description of $\mathfrak{b}^{\left[1 / p^{e}\right]}$, when $\mathfrak{b}$ is an arbitrary ideal on $X$. Suppose that $X$ is an irreducible, smooth scheme of finite type over a perfect field $L$ of characteristic $p$. Let $\omega_{X}=\wedge^{n} \Omega_{X / L}$, where $n=\operatorname{dim}(X)$. Recall that the Cartier isomorphism (see [Deligne and Illusie 1987]) gives in particular an isomorphism $\omega_{X} \simeq \mathscr{H}^{n}\left(F_{*} \Omega_{X / L}^{\bullet}\right)$, where $F$ is the (absolute) Frobenius morphism, and $\Omega_{X / L}^{\bullet}$ is the de Rham complex of $X$. In particular, we get a surjective $O_{X}$-linear map $t_{X}: F_{*} \omega_{X} \rightarrow \omega_{X}$. This can be explicitly described in coordinates, as follows. Suppose that $u_{1}, \ldots, u_{n} \in \mathcal{O}_{X, x}$ form a regular system of parameters, where $x \in X$ is a closed point. We may assume that $u_{1}, \ldots, u_{n}$ are defined in an affine open neighborhood $U$ of $x$, and that $d u=d u_{1} \wedge \cdots \wedge d u_{n}$ gives a basis of $\omega_{X}$ on $U$. Furthermore, we may assume that $\mathcal{O}_{U}$ is free over $\mathcal{O}_{U}^{p}$, with basis

$$
\left\{u_{1}^{i_{1}} \cdots u_{n}^{i_{n}} \mid 0 \leq i_{j} \leq p-1 \text { for } 1 \leq j \leq n\right\}
$$

(note that the residue field of $\mathrm{O}_{X, x}$ is a finite extension of $L$, hence it is perfect). In this case $t_{X}$ is characterized by the fact that $t_{X}\left(h^{p} w\right)=h \cdot t_{X}(w)$ for every $h \in \mathrm{O}_{X}(U)$, and on the above basis over $\mathrm{O}_{X}(U)^{p}$ it is described by

$$
t_{X}\left(u_{1}^{i_{1}} \cdots u_{n}^{i_{n}} d u\right)= \begin{cases}d u & \text { if } i_{j}=p-1 \text { for all } j \\ 0 & \text { otherwise }\end{cases}
$$

Iterating $e$ times $t_{X}$ gives $t_{X}^{e}: F_{*}^{e} \omega_{X} \rightarrow \omega_{X}$. These maps are functorial in the following sense. If $\pi: Y \rightarrow X$ is a proper birational morphism between irreducible 
smooth varieties as above, then we have a commutative diagram

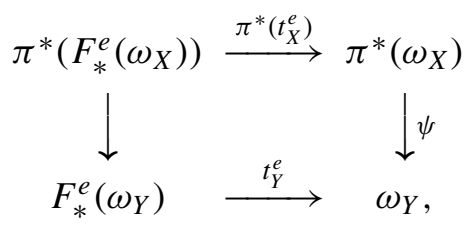

where $\psi$ is the canonical morphism induced by pulling-back $n$-forms, and the left vertical map is the composition

$$
\pi^{*}\left(F_{*}^{e}\left(\omega_{X}\right)\right) \longrightarrow F_{*}^{e}\left(\pi^{*}\left(\omega_{X}\right)\right) \stackrel{F_{*}^{e}(\psi)}{\longrightarrow} F_{*}^{e}\left(\omega_{Y}\right) .
$$

Suppose now that $X$ is as above, and $\mathfrak{b}$ is an ideal on $X$. Since $\omega_{X}$ is a line bundle, it follows that the image of $F_{*}^{e}\left(\mathfrak{b} \cdot \omega_{X}\right)$ by $t_{X}^{e}$ can be written as $J \cdot \omega_{X}$ for a unique ideal $J$ on $X$. It is an easy consequence of the description of $\mathfrak{b}^{\left[1 / p^{e}\right]}$ in [Blickle et al. 2008, Proposition 2.5] and of formula (4) that in fact $J=\mathfrak{b}^{\left[1 / p^{e}\right]}$; see also [Blickle et al. 2010, Proposition 3.10].

\section{A review of nonstandard constructions}

We begin by reviewing some general facts about ultraproducts. For a detailed introduction to this topic, the reader is referred to [Goldblatt 1998]. We then explain how geometric objects over an ultraproduct of fields correspond to sequences of such geometric objects over the fields we are starting with, up to a suitable equivalence relation. Most of this material is well-known to the experts, and can be found, for example, in [Schoutens 2005, §2]. However, we prefer to give a detailed presentation for the benefit of those readers having little or no familiarity with nonstandard constructions.

3A. Ultrafilters and ultraproducts. Recall that an ultrafilter on the set of positive integers $\mathbb{N}$ is a nonempty collection $\mathcal{U}$ of subsets of $\mathbb{N}$ that satisfies the following properties:

(i) If $A$ and $B$ lie in $U$, then $A \cap B$ lies in $U$.

(ii) If $A \subseteq B$ and $A$ is in $U$, then $B$ is in $U$.

(iii) The empty set does not belong to $\mathcal{U}$.

(iv) Given any $A \subseteq \mathbb{N}$, either $A$ or $\mathbb{N} \backslash A$ lies in $U$.

An ultrafilter $\mathcal{U}$ is nonprincipal if no finite subsets of $\mathbb{N}$ lie in $\mathcal{U}$. It is an easy consequence of Zorn's Lemma that nonprincipal ultrafilters exist, and we fix one such ultrafilter $U$. Given a property $\mathscr{P}(m)$, where $m \in \mathbb{N}$, we say that $\mathscr{P}(m)$ holds for almost all $m$ if $\{m \in \mathbb{N} \mid \mathscr{P}(m)$ holds $\}$ lies in $U$. 
Given a sequence of sets $\left(A_{m}\right)_{m \in \mathbb{N}}$, the ultraproduct $\left[A_{m}\right]$ is the quotient of $\prod_{m \in \mathbb{N}} A_{m}$ by the equivalence relation given by $\left(a_{m}\right) \sim\left(b_{m}\right)$ if $a_{m}=b_{m}$ for almost all $m$. We write the class of $\left(a_{m}\right)$ in $\left[A_{m}\right]$ by $\left[a_{m}\right]$. Note that the element $\left[a_{m}\right]$ is well-defined even if $a_{m}$ is only defined for almost all $m$. Similarly, the set [ $\left.A_{m}\right]$ is well-defined if we give $A_{m}$ for almost all $m$.

If $A_{m}=A$ for all $m$, then one writes ${ }^{*} A$ instead of $\left[A_{m}\right]$. This is the nonstandard extension of $A$. Note that there is an obvious inclusion $A \hookrightarrow{ }^{*} A$ that takes $a \in A$ to the class of the constant sequence $(a)$.

The general principle is that if all $A_{m}$ have a certain algebraic structure, then so does $\left[A_{m}\right]$, by defining the corresponding structure component-wise on $\prod_{m \in \mathbb{N}} A_{m}$. For example, if we consider fields $\left(L_{m}\right)_{m \in \mathbb{N}}$, then $k:=\left[L_{m}\right]$ is a field. In particular, the nonstandard extension ${ }^{*} \mathbb{R}$ of $\mathbb{R}$ is an ordered field. Furthermore, it is easy to see that if all $L_{m}$ are algebraically closed, then so is $k$. Note also that if $\lim _{m \rightarrow \infty} \operatorname{char}\left(L_{m}\right)=\infty$, then $\operatorname{char}(k)=0$.

Given a sequence of maps $f_{m}: A_{m} \rightarrow B_{m}$ for $m \in \mathbb{N}$, we get a map $\left[f_{m}\right]$ : $\left[A_{m}\right] \rightarrow\left[B_{m}\right]$ that takes $\left[a_{m}\right]$ to $\left[f_{m}\left(a_{m}\right)\right]$. In particular, given a map $f: A \rightarrow B$, we get a map ${ }^{*} f:{ }^{*} A \rightarrow{ }^{*} B$ that extends $f$. If each $A_{m}$ is a subset of $B_{m}$, we can identify $\left[A_{m}\right]$ to a subset of $\left[B_{m}\right]$ via the corresponding map. The subsets of $\left[B_{m}\right]$ of this form are called internal.

We will use in Section 4 the following notion. Suppose that $u=\left[u_{m}\right] \in \mathbb{R}$ is bounded (this means that there is $M \in \mathbb{R}_{>0}$ such that $* u \mid \leq M$, that is, $\left|u_{m}\right| \leq M$ for almost all $m$ ). In this case, there is a unique real number, the $\operatorname{shadow} \operatorname{sh}(u)$ of $u$, with the property that for every positive real number $\varepsilon$, we have $*|u-\operatorname{sh}(u)|<\varepsilon$, that is, $\left|\operatorname{sh}(u)-u_{m}\right|<\varepsilon$ for almost all $m$. We refer to [Goldblatt 1998, §5.6] for a discussion of shadows. A useful property is that if $\left(c_{m}\right)_{m \in \mathbb{N}}$ is a convergent sequence, with $\lim _{m \rightarrow \infty} c_{m}=c$, then $\operatorname{sh}\left(\left[c_{m}\right]\right)=c$; see [ibid., Theorem 6.1]. On the other hand, it is a consequence of the definition that $\operatorname{sh}\left(\left[c_{m}\right]\right)$ is the limit of a suitable subsequence of $\left(c_{m}\right)_{m \in \mathbb{N}}$.

\section{B. Schemes, morphisms, and sheaves over an ultraproduct of fields. Suppose} that $U$ is a nonprincipal ultrafilter on $\mathbb{N}$ as in the previous section, and suppose that $\left(L_{m}\right)_{m \in \mathbb{N}}$ is a sequence of fields. We denote the corresponding ultraproduct by $k=\left[L_{m}\right]$. Let us temporarily fix $n \geq 1$, and consider the polynomial rings $R_{m}=L_{m}\left[x_{1}, \ldots, x_{n}\right]$. We write $k\left[x_{1}, \ldots, x_{n}\right]_{\text {int }}$ for the ring $\left[R_{m}\right]$, the ring of internal polynomials in $n$ variables (we emphasize, however, that the elements of this ring are not polynomials). Given a sequence of ideals $\left(\mathfrak{a}_{m} \subseteq R_{m}\right)_{m \in \mathbb{N}}$, we get the internal ideal $\left[\mathfrak{a}_{m}\right]$ in $k\left[x_{1}, \ldots, x_{n}\right]_{\text {int }}$.

We have an embedding $k\left[x_{1}, \ldots, x_{n}\right] \hookrightarrow k\left[x_{1}, \ldots, x_{n}\right]_{\text {int }}$. Its image consists of those $g=\left[g_{m}\right] \in k\left[x_{1}, \ldots, x_{n}\right]_{\text {int }}$ for which there is $d \in \mathbb{N}$ such that $\operatorname{deg}\left(g_{m}\right) \leq d$ for almost all $m$ (in this case we say that $g$ has bounded degree). We say that an 
ideal $\mathfrak{b} \subseteq k\left[x_{1}, \ldots, x_{n}\right]_{\text {int }}$ is generated in bounded degree if it is generated by an ideal in $k\left[x_{1}, \ldots, x_{n}\right]$ (in which case $\mathfrak{b}$ is automatically an internal ideal). Given an ideal $\mathfrak{a}$ in $k\left[x_{1}, \ldots, x_{n}\right]$, we put $\mathfrak{a}_{\text {int }}:=\mathfrak{a} \cdot k\left[x_{1}, \ldots, x_{n}\right]_{\text {int }}$.

The connection between $k\left[x_{1}, \ldots, x_{n}\right]$ and $k\left[x_{1}, \ldots, x_{n}\right]_{\text {int }}$ is studied in [van den Dries and Schmidt 1984]. In particular:

Theorem 3.1 [van den Dries and Schmidt 1984, Theorem 1.1]. The extension $k\left[x_{1}, \ldots, x_{n}\right] \hookrightarrow k\left[x_{1}, \ldots, x_{n}\right]_{\text {int }}$ is faithfully flat. In particular, given any ideal $\mathfrak{a}$ in $k\left[x_{1}, \ldots, x_{n}\right]$, we have $\mathfrak{a}_{\text {int }} \cap k\left[x_{1}, \ldots, x_{n}\right]=\mathfrak{a}$.

It follows from the theorem that ideals of $k\left[x_{1}, \ldots, x_{n}\right]_{\text {int }}$ generated in bounded degree are in order-preserving bijection with the ideals in $k\left[x_{1}, \ldots, x_{n}\right]$. Furthermore, note that every such ideal of $k\left[x_{1}, \ldots, x_{n}\right]_{\text {int }}$ is of the form $\left[\mathfrak{a}_{m}\right]$ for a sequence $\left(\mathfrak{a}_{m}\right)_{m \in \mathbb{N}}$ that is generated in bounded degree, that is, such that for some $d, \mathfrak{a}_{m} \subseteq L_{m}\left[x_{1}, \ldots, x_{n}\right]$ is generated by polynomials of degree less than or equal to $d$ for almost all $m$. Of course, we have $\left[\mathfrak{a}_{m}\right]=\left[\mathfrak{b}_{m}\right]$ if and only $\mathfrak{a}_{m}=\mathfrak{b}_{m}$ for almost all $m$. Given such a sequence $\left(\mathfrak{a}_{m}\right)_{m \in \mathbb{N}}$, we call $\left[\mathfrak{a}_{m}\right] \cap k\left[x_{1}, \ldots, x_{n}\right]$ the ideal of polynomials corresponding to the sequence.

Our next goal is to describe how to associate to a geometric object over $k$ a sequence of corresponding objects over each of $L_{m}$ (in fact, an equivalence class of such sequences). Given a separated scheme $X$ of finite type over $k$, we will associate to it an internal scheme $\left[X_{m}\right]$, by which we mean the following: we have schemes $X_{m}$ of finite type over $L_{m}$ for almost all $m$; furthermore, two such symbols $\left[X_{m}\right]$ and $\left[Y_{m}\right]$ define the same equivalence class if $X_{m}=Y_{m}$ for almost all $m$. An internal morphism $\left[f_{m}\right]:\left[X_{m}\right] \rightarrow\left[Y_{m}\right]$ between internal schemes consists of an equivalence class of sequences of morphisms of schemes $f_{m}: X_{m} \rightarrow Y_{m}$ (defined for almost all $m$ ), where $\left[f_{m}\right]=\left[g_{m}\right]$ if $f_{m}=g_{m}$ for almost all $m$.

We want to define a functor $X \rightarrow X_{\text {int }}$ from separated schemes of finite type over $k$ to internal schemes. We first consider the case when $X$ is affine. In this case let us choose a closed embedding $X \hookrightarrow \mathbb{A}_{k}^{N}$, defined by the ideal $\mathfrak{a} \subseteq k\left[x_{1}, \ldots, x_{N}\right]$. If $\mathfrak{a}_{\text {int }}=\left[\mathfrak{a}_{m}\right]$, then we take $X_{m}$ to be defined in $\mathbb{A}_{L_{m}}^{N}$ by $\mathfrak{a}_{m}$. Note that $X_{\text {int }}:=\left[X_{m}\right]$ is well defined. We also put $\mathcal{O}(X)_{\text {int }}:=\left[L_{m}\left[x_{1}, \ldots, x_{N}\right] / \mathfrak{a}_{m}\right]$, and note that we have a canonical ring homomorphism $\eta_{X}: \mathcal{O}(X) \rightarrow \mathcal{O}(X)_{\text {int }}$. Suppose now that we have a morphism $f: Y \rightarrow X$ of affine schemes as above, and closed embeddings $Y \hookrightarrow \mathbb{A}_{k}^{N}$ and $X \hookrightarrow \mathbb{A}_{k}^{M}$. We have a homomorphism $\varphi: k\left[x_{1}, \ldots, x_{M}\right] \rightarrow k\left[x_{1}, \ldots, x_{N}\right]$ that induces $f$, and that extends to an internal morphism $k\left[x_{1}, \ldots, x_{M}\right]_{\text {int }} \rightarrow$ $k\left[x_{1}, \ldots, x_{N}\right]_{\text {int }}$. This induces morphisms $f_{m}: Y_{m} \rightarrow X_{m}$ for almost all $m$, hence an internal morphism $Y_{\text {int }} \rightarrow X_{\text {int }}$. It is easy to see that this is independent of the choice of the lifting $\varphi$ and that it is functorial.

The first consequence is that if we replace $X \hookrightarrow \mathbb{A}_{k}^{N}$ by a different embedding $X \hookrightarrow \mathbb{A}_{k}^{M}$, then the two internal schemes that we obtain are canonically isomorphic. 
We use this to extend the above definition to the case when $X$ is not necessarily affine, as follows. Note first that if $\overline{L_{m}}$ is an algebraic closure of $L_{m}$, and if $\bar{k}=\left[\overline{L_{m}}\right]$, then $\bar{k}$ is an algebraically closed field containing $k$, and for every affine $X$ as above, with $X_{\text {int }}=\left[X_{m}\right]$, we have a natural bijection of sets $X(\bar{k}) \simeq\left[X_{m}\left(\overline{L_{m}}\right)\right]$.

Lemma 3.2. Let $X$ be an affine scheme as above, $U \subset X$ an affine open subset, and write $X_{\mathrm{int}}=\left[X_{m}\right]$ and $U_{\mathrm{int}}=\left[U_{m}\right]$.

(i) The induced maps $U_{m} \rightarrow X_{m}$ are open immersions for almost all $m$.

(ii) If $X=U_{1} \cup \cdots \cup U_{r}$ is an affine open cover, and $\left(U_{i}\right)_{\mathrm{int}}=\left[U_{i, m}\right]$ for every $i$, then $X_{m}=U_{1, m} \cup \cdots \cup U_{r, m}$ for almost all $m$.

Proof. The first assertion is clear in the case when $U$ is a principal affine open subset corresponding to $f \in \mathscr{O}(X)$ : if the image of $f$ in $\mathcal{O}(X)_{\text {int }}$ is $\left[f_{m}\right]$, then for almost all $m$ we have that $U_{m}$ is the principal affine open subset of $X_{m}$ corresponding to $U_{m}$. The assertion in (ii) is clear, too, when all $U_{i}$ are principal affine open subsets in $X$ : once we know that the $U_{i, m}$ are open in $X_{m}$, to get the assertion we want it is enough to look at the $\bar{k}$-valued points of $X$.

We now obtain the assertion in (i) in general, since we may cover $U$ by finitely many principal affine open subsets in $X$ (hence also in $U$ ). We then deduce (ii) in general from (i) by considering the $\bar{k}$-valued points of $X$.

Given any scheme $X$, separated and of finite type over $k$, consider an affine open cover $X=U_{1} \cup \cdots \cup U_{r}$, and let $\left(U_{i}\right)_{\text {int }}=\left[U_{i, m}\right]$. The intersection $U_{i} \cap U_{j}$ is affine and open in both $U_{i}$ and $U_{j}$, hence by Lemma 3.2, $U_{i, m} \cap U_{j, m}$ is affine and open in both $U_{i, m}$ and $U_{j, m}$ for almost all $m$. We get $X_{m}$ by gluing, for all $i$ and $j$, the open subsets $U_{i, m}$ and $U_{j, m}$ along $\left(U_{i} \cap U_{j}\right)_{m}$, and put $X_{\text {int }}=\left[X_{m}\right]$. It is straightforward to check that $X_{\text {int }}$ is independent of the choice of cover (up to a canonical isomorphism). Similarly, given a morphism of schemes $f: Y \rightarrow X$ we get an internal morphism $f_{\text {int }}=\left[f_{m}\right]: Y_{\text {int }} \rightarrow X_{\text {int }}$ by gluing the internal morphisms obtained by restricting $f$ to suitable affine open subsets. Therefore we have a functor from the category of separated schemes of finite type over $k$ to the category of internal schemes and internal morphisms. This has the property that given $L_{m}$-algebras $A_{m}$ for almost all $m$, if $A=\left[A_{m}\right]$, then we have a natural bijection of sets

$$
\operatorname{Hom}(\operatorname{Spec} A, X) \simeq\left[\operatorname{Hom}\left(\operatorname{Spec} A_{m}, X_{m}\right)\right],
$$

where $X_{\text {int }}=\left[X_{m}\right]$. In particular, we have a bijection $X(\bar{k}) \simeq\left[X_{m}\left(\overline{L_{m}}\right)\right]$.

We do not attempt to give a comprehensive account of the properties of this construction, but list in the following proposition a few that we will need.

Proposition 3.3. Let $X$ and $Y$ be separated schemes of finite type over $k$, and $X_{\mathrm{int}}=\left[X_{m}\right]$ and $Y_{\mathrm{int}}=\left[Y_{m}\right]$ the corresponding internal schemes. 
(i) For every affine open subset $U$ of $X$, the ring homomorphism

$$
\eta_{U}: \mathrm{O}_{X}(U) \rightarrow \mathrm{O}_{X}(U)_{\text {int }}
$$

is faithfully flat.

(ii) $X$ is reduced or integral if and only if $X_{m}$ has the same property for almost all $m$.

(iii) The internal scheme corresponding to $X \times Y$ is $\left[X_{m} \times Y_{m}\right]$.

(iv) If $f: Y \rightarrow X$ is an open or closed immersion, then the induced morphisms $f_{m}: Y_{m} \rightarrow X_{m}$ have the same property for almost all $m$. In particular, $X_{m} i s$ separated for almost all $m$.

(v) If $X^{(1)}, \ldots, X^{(r)}$ are the irreducible components of $X$, and $X_{\mathrm{int}}^{(i)}=\left[X_{m}^{(i)}\right]$, then $X_{m}^{(1)}, \ldots, X_{m}^{(r)}$ are the irreducible components of $X_{m}$ for almost all $m$.

(vi) If $X$ is affine and $f: Y \rightarrow X$ is a projective morphism, then $f_{m}: Y_{m} \rightarrow X_{m}$ is projective for almost all $m$.

(vii) If $\operatorname{dim}(X)=d$, then $\operatorname{dim}\left(X_{m}\right)=d$ for almost all $m$.

Proof. The assertion in (i) follows from definition and Theorem 3.1. The assertions in (ii) follow from definition and the fact that if $\mathfrak{a}$ is an ideal in $k\left[x_{1}, \ldots, x_{N}\right]$, then $\mathfrak{a}$ is prime or radical if and only if $\mathfrak{a} \cdot k\left[x_{1}, \ldots, x_{n}\right]_{\text {int }}$ has the same property; see [van den Dries and Schmidt 1984, Theorem 2.5, Corollary 2.7]. Properties (iii) and (iv) are easy consequences of the definition (note that we have already checked the assertion regarding open immersions when both $X$ and $Y$ are affine). The second assertion in (iv) follows from the fact that the diagonal map $X \rightarrow X \times X$ being a closed immersion implies that $X_{m} \rightarrow X_{m} \times X_{m}$ is a closed immersion for almost all $m$. We obtain (v) from (ii), (iv), and the fact that $X_{m}^{(1)}, \ldots, X_{m}^{(r)}$ cover $X_{m}$ for almost all $m$. This follows by computing the $\bar{k}$-points of $X$, and using (6).

In order to prove (vi), note that $\left(\mathbb{P}_{k}^{N}\right)_{\text {int }} \simeq\left[\mathbb{P}_{L_{m}}^{N}\right]$. Therefore a closed embedding $\iota: Y \hookrightarrow X \times \mathbb{P}_{k}^{N}$ induces by (iii) and (iv) closed embeddings $\iota_{m}: Y_{m} \hookrightarrow X_{m} \times \mathbb{P}_{L_{m}}^{N}$ for almost all $m$.

We prove (vii) by induction on $\operatorname{dim}(X)$. Using (v), we reduce to the case when $X$ is irreducible. After replacing $X$ by $X_{\text {red }}$, we see that we may assume, in fact, that $X$ is integral, hence by (ii), for almost all $m$ we have $X_{m}$ integral. It is enough to prove the assertion for an affine open subset $U$ of $X$, hence we may assume that $X=\operatorname{Spec} A$ is affine, and let us write $X_{m}=\operatorname{Spec} A_{m}$. If $f \in \mathcal{O}(X)$ is nonzero, then $\operatorname{dim}(A /(f))=\operatorname{dim}(A)-1$. Let $\left[f_{m}\right]=\eta_{X}(f) \in \mathcal{O}(X)_{\text {int }}$, hence for almost all $m$ we have $f_{m} \neq 0$ and $\operatorname{dim}\left(A_{m} /\left(f_{m}\right)\right)=\operatorname{dim}\left(A_{m}\right)-1$. Since the internal scheme corresponding to $\operatorname{Spec} A /(f)$ is [Spec $A_{m} /\left(f_{m}\right)$ ], we conclude by induction. 
Remark 3.4. We emphasize that to an arbitrary internal scheme $\left[X_{m}\right]$ we do not associate a scheme over $k$. In order to illustrate the problems that arise when trying to do this, consider the following two examples.

1) Let $X_{m}=\operatorname{Spec}\left(R_{m}\right)$, where $R_{m}=L_{m}\left[x_{1}, \ldots, x_{m}\right]$.

2) Let $Y_{m}$ be the closed subscheme of $\operatorname{Spec}\left(L_{m}[x, y]\right)$ defined by $\left(f_{m}\right)$, where $f_{m}=x^{2}+y^{m}$.

The only reasonable schemes to associate to $\left[X_{m}\right]$ and $\left[Y_{m}\right]$ are $X=\operatorname{Spec}\left(\left[R_{m}\right]\right)$ and $Y=\operatorname{Spec}\left(k[x, y]_{\text {int }} /(f)\right)$, respectively, where $f=\left[f_{m}\right]=x^{2}+y^{\omega}$, with $\omega$ being the nonstandard integer corresponding to $(1,2,3, \ldots)$. In this case, the internal $k$-valued points of $X$ and $Y$ are in natural bijection with $\left[X_{m}\left(L_{m}\right)\right]$ and $\left[Y_{m}\left(L_{m}\right)\right]$, respectively. However, since both $X$ and $Y$ are far from being of finite type over $k$, we will not further consider such general constructions.

Suppose now that $X$ is a scheme over $k$ as above, and $\mathscr{F}$ is a coherent sheaf on $X$. If $X_{\text {int }}=\left[X_{m}\right]$, we define an internal coherent sheaf on $\left[X_{m}\right]$ to be a symbol [ $\left.\mathscr{F}_{m}\right]$, where $\mathscr{F}_{m}$ is defined for almost all $m$ and is a coherent sheaf of $X_{m}$. Furthermore, two such symbols $\left[\mathscr{F}_{m}\right]$ and $\left[\mathscr{F}_{m}^{\prime}\right]$ are identified precisely when $\mathscr{F}_{m}=\mathscr{F}_{m}^{\prime}$ for almost all $m$. A morphism of internal coherent sheaves is defined in a similar way, and we get an abelian category consisting of internal coherent sheaves on $\left[X_{m}\right]$.

We now define a functor $\mathscr{F}_{F} \rightarrow \mathscr{F}_{\text {int }}$ from the category of coherent sheaves on $X$ to that of internal coherent sheaves on $X_{\text {int }}$. Given an affine open subset $U$ of $X$ and the corresponding internal scheme $U_{\text {int }}=\left[U_{m}\right]$, we consider the $\mathscr{O}_{X}(U)_{\text {int }}$-module $\mathscr{T}_{U}:=\mathscr{F}(U) \otimes_{\mathscr{O}_{X}(U)} \mathscr{O}_{X}(U)_{\text {int }}$. We claim that this is equal to $\left[M_{m}\right]$ for suitable $\mathrm{O}_{X_{m}}\left(U_{m}\right)$-modules $M_{m}$. Indeed, this follows by considering a finite free presentation

$$
\mathrm{O}_{X}(U)^{\oplus r} \stackrel{\varphi}{\rightarrow} \mathrm{O}_{X}(U)^{\oplus s} \rightarrow \mathscr{F}(U) \rightarrow 0 .
$$

If $\varphi$ is defined by a matrix $\left(a_{i, j}\right)_{i, j}$ and if we write $\eta_{U}\left(a_{i, j}\right)=\left[a_{i, j, m}\right]$, then we may take each $M_{m}$ to be the cokernel of the map $\mathcal{O}_{X_{m}}\left(U_{m}\right)^{\oplus r} \rightarrow \mathcal{O}_{X_{m}}\left(U_{m}\right)^{\oplus s}$ defined by the matrix $\left(a_{i, j, m}\right)_{i, j}$. We put $\mathscr{F}_{m}(U)=M_{m}$ for almost all $m$. It is now easy to see that the $\mathscr{F}_{m}(U)$ glue together for almost all $m$ to give coherent sheaves $\mathscr{F}_{m}$ on $X_{m}$. Therefore we get an internal coherent sheaf $\mathscr{F}_{\text {int }}$ on $X_{\text {int }}$. Given a morphism of coherent sheaves on $X$, we clearly get a corresponding morphism of internal coherent sheaves. It follows from definition and Proposition 3.3 (i) that this functor is exact in a strong sense: a bounded complex of coherent sheaves on $X$ is acyclic if and only if the corresponding complexes of coherent sheaves on $X_{m}$ are acyclic for almost all $\mathrm{m}$. Note also that the functor is compatible with tensor product: if $\mathscr{F}_{\text {int }}=\left[\mathscr{F}_{m}\right]$ and $\mathscr{G}_{\text {int }}=\left[\mathscr{G}_{m}\right]$, then $\left(\mathscr{F}_{\otimes_{X}} \mathscr{G}_{\mathcal{O}_{\text {int }}}\right.$ is canonically isomorphic to $\left[\mathscr{F}_{m} \otimes_{\mathbb{O}_{X}} \mathscr{G}_{m}\right]$. We collect in the following proposition a few other properties of this functor that we will need. 
Proposition 3.5. Let $X$ be a separated scheme of finite type over $k$, and F a coherent sheaf on X. Consider $X_{\mathrm{int}}=\left[X_{m}\right]$ and $\mathscr{F}_{\mathrm{int}}=\left[\mathscr{F}_{m}\right]$.

(i) $\mathscr{F}_{F}$ is locally free of rank $r$ if and only if $\mathscr{F}_{m}$ has the same property for almost all $m$.

(ii) If $\mathscr{F}_{F}$ is an ideal in $\mathrm{O}_{X}$ defining the closed subscheme $Z$ of $X$, and $Z_{\text {int }}=\left[Z_{m}\right]$, then $\mathscr{F}_{m}$ is (isomorphic to) the ideal defining $Z_{m}$ in $X_{m}$ for almost all $m$.

(iii) If $f: Y \rightarrow X$ is a morphism of schemes as above, and $f_{\mathrm{int}}=\left[f_{m}\right]$, then we have a canonical isomorphism $f^{*}(\mathscr{F})_{\mathrm{int}} \simeq\left[f_{m}^{*}\left(\mathscr{F}_{m}\right)\right]$.

(iv) If $g: Y \rightarrow X$ is a projective morphism of schemes as above, and $g_{\mathrm{int}}=$ $\left[g_{m}\right]:\left[Y_{m}\right] \rightarrow\left[X_{m}\right]$, then for every $i \geq 0$ we have a canonical isomorphism

$$
R^{i} f_{*}(\mathscr{F})_{\mathrm{int}} \simeq\left[R^{i}\left(f_{m}\right)_{*}\left(\mathscr{F}_{m}\right)\right] .
$$

(v) If $f$ is as in (iv), $X$ is affine, and $\mathscr{F}$ is a line bundle on $X$ that is (very) ample over $X$, then $\mathscr{F}_{m}$ is (very) ample over $X_{m}$ for almost all $m$.

Proof. The first assertion follows from Proposition 3.3 (i) and the fact that given a faithfully flat ring homomorphism $A \rightarrow B$, a finitely generated $A$-module $M$ is locally free of rank $r$ if and only if the $B$-module $M \otimes_{A} B$ is locally free of rank $r$. Assertion (ii) is an immediate consequence of the definitions. In order to prove (iii) it is enough to consider the case when both $X$ and $Y$ are affine. In this case the assertion follows from the natural isomorphism $\left[M_{m}\right] \otimes_{\left[A_{m}\right]}\left[B_{m}\right] \simeq\left[M_{m} \otimes_{A_{m}} B_{m}\right]$ whenever $A_{m} \rightarrow B_{m}$ are ring homomorphisms and the $M_{m}$ are finitely generated $A_{m}$-modules.

Let us now prove (iv). Suppose first that $X$ is affine. The first step is to construct canonical morphisms

$$
H^{i}\left(Y, \mathscr{F}_{i}\right)_{\mathrm{int}} \rightarrow\left[H^{i}\left(Y_{m}, \mathscr{F}_{m}\right)\right] .
$$

This can be done by computing the cohomology as Čech cohomology with respect to a finite affine open cover of $Y$, and the corresponding affine open covers of $Y_{m}$ (and by checking that the definition is independent of the cover). It is enough to prove that the maps (7) are isomorphisms: if $X$ is not affine, then we simply glue the corresponding isomorphisms over a suitable affine open cover of $X$. Since $Y$ is isomorphic to a closed subscheme of some $X \times \mathbb{P}_{k}^{N}$, it is enough to prove that the morphisms (7) are isomorphisms when $Y=\mathbb{P}_{X}^{N}$. Explicit computation of cohomology implies that (7) is an isomorphism when $\mathscr{F}=\mathbb{O}_{\mathbb{P}_{X}^{N}}(\ell)$ (note that $\left.\mathcal{O}_{\mathbb{P}_{X}^{N}}(\ell)_{\text {int }} \simeq\left[\mathcal{O}_{\mathbb{P}_{X_{m}}^{N}}(\ell)\right]\right)$.

We now prove that (7) is an isomorphism by descending induction on $i$, the case $i>N$ being trivial. Given any $\mathscr{F}$, there is an exact sequence

$$
0 \rightarrow \mathscr{G} \rightarrow \mathbb{O}_{\mathbb{P}_{X}^{N}}(\ell)^{\oplus r} \rightarrow \mathscr{F} \rightarrow 0,
$$


for some $\ell$ and $r$. We use the induction hypothesis, the long exact sequence in cohomology and the 5-lemma to show first that (7) is surjective for all $\mathscr{F}$. Applying this for $\mathscr{G}$, we then conclude that (7) is also injective for all $\mathscr{F}$. This completes the proof of (iv). The assertion in (v) follows using (iii) and Proposition 3.3 (iv), from the fact that if $Y=\mathbb{P}_{X}^{N}$ and $\mathscr{F}_{F}=\mathcal{O}_{Y}(1)$, then $Y_{m} \simeq \mathbb{P}_{X_{m}}^{N}$ and $\mathscr{F}_{m} \simeq \mathcal{O}_{Y_{m}}(1)$ for almost all $m$.

We will need the following uniform version of asymptotic Serre vanishing; see also [Schoutens 2005, Corollary 2.16].

Corollary 3.6. Let $f: Y \rightarrow X$ be a projective morphism of schemes over $k$ as above, with $X$ affine. If $\mathscr{F}$ is a coherent sheaf on $Y$ and $\mathscr{L}$ is a line bundle on $Y$ that is ample over $X$ and such that $H^{i}\left(Y, \mathscr{F} \otimes \mathscr{L}^{j}\right)=0$ for all $i \geq 1$ and all $j \geq j_{0}$, then for almost all $m$ we have $H^{i}\left(Y_{m}, \mathscr{F}_{m} \otimes \mathscr{L}_{m}^{j}\right)=0$ for all $i \geq 1$ and $j \geq j_{0}$, where $Y_{\text {int }}=\left[Y_{m}\right], \mathscr{F}_{\text {int }}=\left[\mathscr{F}_{m}\right]$, and $\mathscr{L}_{\text {int }}=\left[\mathscr{L}_{m}\right]$.

Proof. Note first that we may assume that $\mathscr{L}$ is very ample. Indeed, if $N$ is such that $\mathscr{L}^{N}$ is very ample, then we may apply the very ample case to the line bundle $\mathscr{L}^{N}$ and to the sheaves $\mathscr{F}, \mathscr{F} \otimes \mathscr{L}, \ldots, \mathscr{F} \otimes \mathscr{L}^{N-1}$ to obtain the assertion in the corollary. Let $r=\operatorname{dim}(Y)$. It follows from Proposition 3.5 (iv) that if $i \geq 1$ and $j \geq j_{0}$ are fixed, then $H^{i}\left(Y_{m}, \mathscr{F}_{m} \otimes \mathscr{L}_{m}^{j}\right)=0$ for almost all $m$. In particular, for almost all $m$ we have $H^{i}\left(Y_{m}, \mathscr{F}_{m} \otimes \mathscr{L}_{m}^{j}\right)=0$ for $1 \leq i \leq \operatorname{dim}\left(Y_{m}\right)=r$ and $j_{0} \leq j \leq j_{0}+r-1$. For every such $m$, it follows that $\mathscr{F}_{m}$ is $\left(j_{0}+r\right)$-regular in the sense of Castelnuovo-Mumford regularity, hence $H^{i}\left(Y_{m}, \mathscr{F}_{m} \otimes \mathscr{L}_{m}^{j}\right)=0$ for every $i \geq 1$ and $j \geq j_{0}+r-i$; see [Lazarsfeld 2004, Chapter 1.8.A]. This completes the proof of the corollary.

Proposition 3.7. If $X$ is a separated scheme of finite type over $k$ and $X_{\mathrm{int}}=\left[X_{m}\right]$, then there is a canonical isomorphism $\left(\Omega_{X / k}\right)_{\mathrm{int}} \simeq\left[\Omega_{X_{m} / L_{m}}\right]$. In particular, $X$ is smooth of pure dimension $n$ if and only if $X_{m}$ is smooth of pure dimension $n$ for almost all $m$.

Proof. It is enough to give a canonical isomorphism $\left(\Omega_{X / k}\right)_{\text {int }}=\left[\Omega_{X_{m} / L_{m}}\right]$ when $X$ is affine. Note that we have such an isomorphism when $X=\mathbb{A}_{k}^{N}$. In general, if $X$ is a closed subscheme of $\mathbb{A}_{k}^{N}$ defined by the ideal $\mathfrak{a}$, the sheaf $\Omega_{X / k}$ is the cokernel of a morphism $\mathfrak{a} /\left.\mathfrak{a}^{2} \rightarrow \Omega_{\mathbb{A}_{k}^{N}}\right|_{X}$. If $\mathfrak{a}=\left[\mathfrak{a}_{m}\right]$, then for almost all $m$ we have an analogous description of each $\Omega_{X_{m} / L_{m}}$ in terms of the embedding $X_{m} \hookrightarrow \mathbb{A}_{L_{m}}^{N}$ given by $\mathfrak{a}_{m}$. Therefore we obtain the desired isomorphism, and one can then check that this is independent of the embedding.

Recall that $X$ is smooth of pure dimension $n$ if and only if $\operatorname{dim}(X)=n$ and $\Omega_{X / k}$ is locally free of rank $n$. The second assertion in the proposition now follows from the first one, together with Proposition 3.3 (vii) and Proposition 3.5 (i).

Suppose that $X$ is a smooth scheme over $k$ as above, and $D=a_{1} D^{(1)}+\cdots+a_{r} D^{(r)}$ is a divisor on $X$. It follows from Proposition 3.3 (ii), (vii) that if $D_{\text {int }}^{(i)}=\left[D_{m}^{(i)}\right]$, 
then $D_{m}^{(i)}$ is a prime divisor on $X_{m}$ for almost all $m$. For all such $m$ we put $D_{m}=a_{1} D_{m}^{(1)}+\cdots+a_{r} D_{m}^{(r)}$.

Remark 3.8. Note that in the case when $D$ is effective, and thus can be considered as a subscheme of $X$, the above convention is compatible with our previous definition via $D_{\text {int }}=\left[D_{m}\right]$. Indeed, if we define the $D_{m}$ via the latter formula, then it follows from definition that since $D$ is locally defined by one nonzero element, the same holds for $D_{m}$ for almost all $m$. Furthermore, Proposition $3.3(\mathrm{v})$ implies that $D_{m}^{(1)}, \ldots, D_{m}^{(r)}$ are the irreducible components of $D_{m}$ for almost all $m$. We also see that the coefficient of $D_{m}^{(i)}$ in $D_{m}$ is equal to $a_{i}$ for almost all $m$ : this follows from the fact that this coefficient is the largest nonnegative integer $d_{i}$ such that $d_{i} D_{m}^{(i)}$ is a subscheme of $D_{m}$.

We thus see that for every divisor $D$, we have $O(D)_{\text {int }}=\left[O\left(D_{m}\right)\right]$. Indeed, when $-D$ is effective, this follows from the above remark and Proposition 3.5 (ii). The general case follows easily by reducing to the case when $X$ is affine, and replacing $D$ by $D+\operatorname{div}(f)$ for a suitable $f \in \mathcal{O}(X)$ such that $-D-\operatorname{div}(f)$ is effective.

Proposition 3.9. Let $X$ be a smooth, separated scheme over $k$, and $D=\sum_{i=1}^{N} D^{(i)}$ an effective divisor on $X$, with simple normal crossings, where the $D^{(i)}$ are distinct prime divisors. If $X_{\mathrm{int}}=\left[X_{m}\right]$ and $D_{\mathrm{int}}=\left[D_{m}\right]$, then $D_{m}$ has simple normal crossings for almost all $m$.

Proof. Note that $X_{m}$ is smooth over $L_{m}$ for almost all $m$ by Proposition 3.7. Since $D$ has simple normal crossings, for every $r$ and every $1 \leq i_{1}<\cdots<i_{r} \leq N$ the subscheme $D^{\left(i_{1}\right)} \cap \cdots \cap D^{\left(i_{r}\right)}$ is smooth over $k$ (possibly empty). It follows from definition that we have

$$
\left(D^{\left(i_{1}\right)} \cap \cdots \cap D^{\left(i_{r}\right)}\right)_{\text {int }}=\left[D_{m}^{\left(i_{1}\right)} \cap \cdots \cap D_{m}^{\left(i_{r}\right)}\right],
$$

hence $D_{m}^{\left(i_{1}\right)} \cap \cdots \cap D_{m}^{\left(i_{r}\right)}$ is smooth over $L_{m}$ for almost all $m$, by another application of Proposition 3.7. Thus $D_{m}$ has simple normal crossings for almost all $m$.

\section{Limits of $F$-pure thresholds}

The following is our main result. As we will see, it easily implies the theorem stated in Section 1.

Theorem 4.1. Let $\left(L_{m}\right)_{m \in \mathbb{N}}$ be a sequence of fields of positive characteristic such that $\lim _{m \rightarrow \infty} \operatorname{char}\left(L_{m}\right)=\infty$. We fix a nonprincipal ultrafilter on $\mathbb{N}$, and let $k=\left[L_{m}\right]$. If $\mathfrak{a}_{m} \subseteq L_{m}\left[x_{1}, \ldots, x_{n}\right]$ are nonzero ideals generated in bounded degree, and if $\mathfrak{a} \subseteq k\left[x_{1}, \ldots, x_{n}\right]$ is the ideal of polynomials corresponding to $\left(\mathfrak{a}_{m}\right)_{m \geq 1}$, then for every $\lambda \in \mathbb{R}_{\geq 0}$ we have

$$
\mathscr{S}\left(\mathfrak{a}^{\lambda}\right)_{\text {int }}=\left[\tau\left(\mathfrak{a}_{m}^{\lambda}\right)\right]
$$


Corollary 4.2. If $\left(\mathfrak{a}_{m}\right)_{m \in \mathbb{N}}$ and $\mathfrak{a}$ are as in the above theorem, and $\mathfrak{a}_{m}$ vanishes at the origin for almost all $m$, then

$$
\operatorname{lct}_{0}(\mathfrak{a})=\operatorname{sh}\left(\left[\operatorname{fpt}_{0}\left(\mathfrak{a}_{m}\right)\right]\right) .
$$

Proof. Note first that since $\mathfrak{a}_{m} \subseteq\left(x_{1}, \ldots, x_{n}\right) L_{m}\left[x_{1}, \ldots, x_{n}\right]$ for almost all $m$, we have $\mathfrak{a} \subseteq\left(x_{1}, \ldots, x_{n}\right) k\left[x_{1}, \ldots, x_{n}\right]$. By definition, we have

$$
\operatorname{lct}_{0}(\mathfrak{a})=\min \left\{\lambda \in \mathbb{R}_{\geq 0} \mid \mathscr{g}\left(\mathfrak{a}^{\lambda}\right) \subseteq\left(x_{1}, \ldots, x_{n}\right)\right\} .
$$

Since $\mathscr{f}\left(\mathfrak{a}^{\lambda}\right) \subseteq\left(x_{1}, \ldots, x_{n}\right)$ if and only if $\mathscr{g}\left(\mathfrak{a}^{\lambda}\right)_{\text {int }} \subseteq\left(x_{1}, \ldots, x_{n}\right)_{\text {int }}$, it follows from Theorem 4.1 that this is the case if and only if $\tau\left(\mathfrak{a}_{m}^{\lambda}\right) \subseteq\left(x_{1}, \ldots, x_{n}\right)$ for almost all $m$. This is further equivalent to $\lambda \geq \operatorname{fpt}_{0}\left(\mathfrak{a}_{m}\right)$ for almost all $m$. We conclude that $\operatorname{lct}_{0}(\mathfrak{a}) \geq \operatorname{fpt}_{0}\left(\mathfrak{a}_{m}\right)$ for almost all $m$. In addition, for every $\varepsilon \in \mathbb{R}_{>0}$, we have $\mathscr{E}\left(\mathfrak{a}^{\text {lct }_{0}(\mathfrak{a})-\varepsilon}\right)_{\text {int }} \nsubseteq\left(x_{1}, \ldots, x_{n}\right)_{\text {int }}$, and using again Theorem 4.1 we deduce that $\tau\left(\mathfrak{a}_{m}^{\text {lct }_{0}(\mathfrak{a})-\varepsilon}\right) \nsubseteq\left(x_{1}, \ldots, x_{n}\right)$ for almost all $m$. By definition, this means that $\operatorname{fpt}_{0}\left(\mathfrak{a}_{m}\right) \geq \operatorname{lct}_{0}(\mathfrak{a})-\varepsilon$ for almost all $m$. This proves the assertion in the corollary.

The result stated in Section 1 is an easy consequence of the above corollary.

Proof of Theorem 1.1. Suppose first that we have a sequence $\left(c_{m}\right)_{m \in \mathbb{N}}$ with $c_{m} \in \mathscr{F}\left(p_{m}\right)_{n}$ for all $m$, and such that $\lim _{m \rightarrow \infty} p_{m}=\infty$ and $c=\lim _{m \rightarrow \infty} c_{m}$. We need to show that $c \in \mathscr{L}_{n}$. By Proposition 2.5, we may assume that there are algebraically closed fields $L_{m}$ of characteristic $p_{m}$, and ideals $\mathfrak{a}_{m} \subseteq L_{m}\left[x_{1}, \ldots, x_{n}\right]$ vanishing at the origin, such that $c_{m}=\operatorname{fpt}_{0}\left(\mathfrak{a}_{m}\right)$. For every $d$, let $\mathfrak{a}_{m}^{(\bar{d})}=\mathfrak{a}_{m}+\left(x_{1}, \ldots, x_{n}\right)^{d}$. It follows from Proposition 2.3 that $\left|\mathrm{fpt}_{0}\left(\mathfrak{a}_{m}\right)-\mathrm{fpt}_{0}\left(\mathfrak{a}_{m}^{(d)}\right)\right| \leq \frac{n}{d}$.

Let $U$ be a nonprincipal ultrafilter on $\mathbb{N}$. We put $k=\left[L_{m}\right]$, and for every $d$, we denote by $\mathfrak{a}^{(d)} \subseteq k\left[x_{1}, \ldots, x_{n}\right]$ the ideal of polynomials associated to the sequence of ideals generated in bounded degree $\left(\mathfrak{a}_{m}^{(d)}\right)_{m \in \mathbb{N}}$. Given any $\varepsilon \in \mathbb{R}_{>0}$, let $d \gg 0$ be such that $\frac{n}{d}<\varepsilon$. By Corollary 4.2, we have $\left|\operatorname{fpt}_{0}\left(\mathfrak{a}_{m}^{(d)}\right)-\operatorname{lct}_{0}\left(\mathfrak{a}^{(d)}\right)\right|<\varepsilon-\frac{n}{d}$ for almost all $m$. Therefore $\left|\operatorname{fpt}_{0}\left(\mathfrak{a}_{m}\right)-\operatorname{lct}_{0}\left(\mathfrak{a}^{(d)}\right)\right|<\varepsilon$ for infinitely many $m$. Since this holds for every $\varepsilon \in \mathbb{R}_{>0}$, we conclude that $c$ lies in the closure of $\left\{\operatorname{lct}_{0}\left(\mathfrak{a}^{(d)}\right) \mid d \geq 1\right\}$. As we have mentioned in Section $2 \mathrm{~A}, \mathscr{L}_{n}$ is closed, hence $c \in \mathscr{L}_{n}$.

In order to prove the converse, let us consider $c \in \mathscr{L}_{n}$. Consider a sequence of prime integers $\left(p_{m}\right)_{m \in \mathbb{N}}$ with limit infinity, and let $L_{m}$ be an algebraically closed field of characteristic $p_{m}$. We fix, as above, a nonprincipal ultrafilter on $\mathbb{N}$, and let $k=\left[L_{m}\right]$. As pointed out in Section $2 \mathrm{~A}$, since $k$ is algebraically closed, we can find an ideal $\mathfrak{b} \subset k\left[x_{1}, \ldots, x_{n}\right]$ vanishing at the origin, such that $c=\operatorname{lct}_{0}(\mathfrak{b})$. Let us write $\mathfrak{b}_{\text {int }}=\left[\mathfrak{b}_{m}\right]$. It follows from Corollary 4.2 that $c$ is the limit of a suitable subsequence of $\left(\mathrm{fpt}_{0}\left(\mathfrak{b}_{m}\right)\right)_{m \in \mathbb{N}}$. This completes the proof of the theorem. Note that the second implication also follows from the results of [Hara and Yoshida 2003] discussed in the introduction. 
Before giving the proof of Theorem 4.1, we describe the approach from [Hara and Yoshida 2003] for proving the equality of multiplier ideals with test ideals in a fixed positive characteristic. The main ingredients are due independently to Hara [1998] and Mehta and Srinivas [1997]. We simplify somewhat the approach in [Hara and Yoshida 2003], avoiding the use of local cohomology, which is important in our nonlocal setting.

Suppose that $L$ is a perfect field of positive characteristic $p$, and $W$ is a smooth, irreducible, $n$-dimensional affine scheme over $L$. We consider a nonzero ideal $\mathfrak{b}$ on $W$, and suppose that we have given a $\log$ resolution $\pi: \widetilde{W} \rightarrow W$ of $\mathfrak{b}$. Let $Z$ be the effective divisor on $\widetilde{W}$ such that $\widetilde{\mathfrak{b}}:=\mathfrak{b} \cdot \mathcal{O}_{\widetilde{W}}=\mathcal{O}_{\widetilde{W}}(-Z)$, and let $E=E_{1}+\cdots+E_{N}$ be a simple normal crossings divisor on $\widetilde{W}$ such that both $K_{\widetilde{W} / W}$ and $Z$ are supported on $E$. For every $\lambda \geq 0$, we put $\mathscr{G}\left(\mathfrak{b}^{\lambda}\right)=\pi_{*} \widetilde{O}_{\widetilde{W}}\left(K_{\widetilde{W} / W}-\lfloor\lambda Z\rfloor\right.$ ) (it is irrelevant for us whether this is independent of the given resolution). In this setting, it is shown in [Hara and Yoshida 2003] that the test ideals are always contained in the multiplier ideals.

Proposition 4.3. With the above notation, we have $\tau\left(\mathfrak{b}^{\lambda}\right) \subseteq \mathscr{g}\left(\mathfrak{b}^{\lambda}\right)$ for all $\lambda \in \mathbb{R}_{\geq 0}$.

Proof. We give a proof using the description of test ideals at the end of Section 2, since the approach will be relevant also when considering the reverse inclusion. We show that

$$
\left(\mathfrak{b}^{m}\right)^{\left[1 / p^{e}\right]} \subseteq \mathscr{J}\left(\mathfrak{b}^{m / p^{e}}\right)
$$

for every $m \geq 0$ and $e \geq 1$. This is enough: given $\lambda \in \mathbb{R}_{\geq 0}$, we have for $e \gg 0$

$$
\tau\left(\mathfrak{b}^{\lambda}\right)=\left(\mathfrak{b}^{\left\lceil\lambda p^{e}\right\rceil}\right)^{\left[1 / p^{e}\right]} \subseteq \mathscr{F}\left(\mathfrak{b}^{\left\lceil\lambda p^{e}\right\rceil / p^{e}}\right)=\mathscr{F}\left(\mathfrak{b}^{\lambda}\right) .
$$

Note that the last equality follows from the fact that $0 \leq\left(\left\lceil\lambda p^{e}\right\rceil / p^{e}\right)-\lambda \ll 1$ for $e \gg 0$.

The commutative diagram (5) induces a commutative diagram

$$
\begin{array}{ccc}
F_{*}^{e}\left(\omega_{W}\right) & \stackrel{t_{W}^{e}}{\longrightarrow} & \omega_{W} \\
\eta=F_{*}^{e}(\rho) \downarrow & \downarrow \rho \\
F_{*}^{e} \pi_{*}\left(\omega_{\widetilde{W}}\right) \stackrel{\pi_{*}\left(t_{\tilde{W}}^{e}\right)}{\longrightarrow} \pi_{*}\left(\omega_{\widetilde{W}}\right),
\end{array}
$$

where the vertical maps are isomorphisms. Note that $t_{\widetilde{W}}^{e}$ induces a (surjective) map $F_{*}^{e}\left(\omega_{\widetilde{W}}(-m Z)\right) \rightarrow \omega_{\widetilde{W}}\left(-\left\lfloor\left(m / p^{e}\right) Z\right\rfloor\right)$, and thus a map

$$
F_{*}^{e} \pi_{*}\left(\omega_{\widetilde{W}}(-m Z)\right) \rightarrow \pi_{*}\left(\omega_{\widetilde{W}}\left(-\left\lfloor\frac{m}{p^{e}} Z\right\rfloor\right)\right) .
$$

Since

$$
\left(\mathfrak{b}^{m}\right)^{\left[1 / p^{e}\right]} \omega_{W}=t_{W}^{e}\left(F_{*}^{e}\left(\mathfrak{b}^{m} \omega_{W}\right)\right)
$$


and $\eta\left(F_{*}^{e}\left(\mathfrak{b}^{m} \omega_{W}\right)\right) \subseteq F_{*}^{e} \pi_{*}\left(\omega_{\widetilde{W}}(-m Z)\right)$, while

$$
\rho^{-1}\left(\pi_{*}\left(\omega_{\widetilde{W}}\left(-\left\lfloor\frac{m}{p^{e}} Z\right\rfloor\right)\right)\right)=\mathscr{g}\left(\mathfrak{b}^{m / p^{e}}\right) \omega_{W},
$$

we see that (8) follows from the fact that $t_{W}^{e} F_{*}^{e}\left(\mathfrak{b}^{m} \omega_{W}\right)=\left(\mathfrak{b}^{m}\right)^{\left[1 / p^{e}\right]} \omega_{W}$ and the commutativity of (9).

We now explain a criterion for the reverse inclusion $\mathscr{g}\left(\mathfrak{b}^{\lambda}\right) \subseteq \tau\left(\mathfrak{b}^{\lambda}\right)$ to hold. We start with the following proposition.

Proposition 4.4. Suppose that $\widetilde{W}$ is a smooth, irreducible, $n$-dimensional variety over the perfect field $L$ of positive characteristic $p$. If $E$ is a simple normal crossings divisor on $\widetilde{W}$, and $G$ is a $\mathbb{Q}$-divisor supported on $E$ such that $-G$ is effective, then the canonical morphism

$$
\Gamma\left(\widetilde{W}, F_{*}^{e}\left(\omega_{\widetilde{W}}\left(\left\lceil p^{e} G\right\rceil\right)\right)\right) \rightarrow \Gamma\left(\widetilde{W}, \omega_{\widetilde{W}}(\lceil G\rceil)\right)
$$

is surjective for every $e \geq 1$, provided that the following two conditions hold:

(A) $H^{i}\left(\widetilde{W}, \Omega_{\widetilde{W}}^{n-i}(\log E)\left(-E+\left\lceil p^{\ell} G\right\rceil\right)\right)=0$ for all $i \geq 1$ and $\ell \geq 1$.

(B) $H^{i+1}\left(\widetilde{W}, \Omega_{\widetilde{W}}^{n-i}(\log E)\left(-E+\left\lceil p^{\ell} G\right\rceil\right)\right)=0$ for all $i \geq 1$ and $\ell \geq 0$.

This is applied as follows. Suppose that $\lambda \in \mathbb{R}_{\geq 0}$ is fixed, and we have a rational number $\mu>\lambda$ such that $\mathscr{F}\left(\mathfrak{b}^{\lambda}\right)=\mathscr{g}\left(\mathfrak{b}^{\mu}\right)$ (note that if $Z=\sum_{i} a_{i} E_{i}$, then it is enough to take $\mu$ such that $\mu-\lambda<\left(\left\lfloor\lambda a_{i}\right\rfloor+1-\lambda a_{i}\right) / a_{i}$ for all $i$ with $\left.a_{i}>0\right)$. Let us consider now a $\mathbb{Q}$-divisor $D$ on $\widetilde{W}$ such that $D$ is ample over $W$, and $-D$ is effective. ${ }^{2}$ We will apply the above proposition with $G=\mu(D-Z)$. We may and will assume that $\lceil G\rceil=\lceil-\mu Z\rceil$ (again this condition only depends on $\mu$ and the coefficients of $Z$; since $-D$ is effective, it is always satisfied if we replace $D$ by $\varepsilon D$, with $0<\varepsilon \ll 1$ ).

Proposition 4.5. With the above notation, if (10) is surjective for every $e \geq 1$, then $\mathscr{g}\left(\mathfrak{b}^{\lambda}\right) \subseteq \tau\left(\mathfrak{b}^{\lambda}\right)$.

Proof. We use again the commutative diagram (9). This induces a commutative diagram

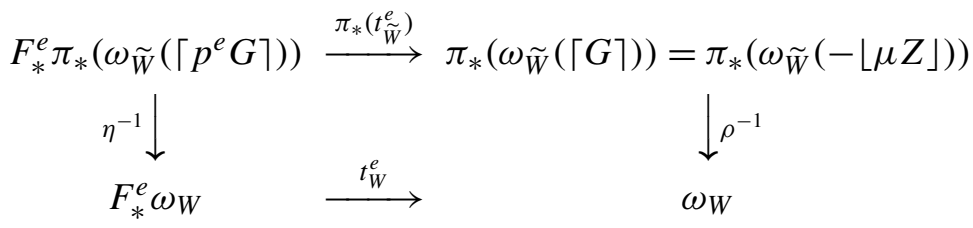

in which the top horizontal map is surjective by assumption (recall that $W$ is affine), and the image of the right vertical map is $\mathscr{f}\left(\mathfrak{b}^{\mu}\right) \omega_{W}$. The image of the left vertical

${ }^{2}$ Such a divisor always exists: if we express $\widetilde{W}$ as the blow-up of $W$ along a suitable ideal, then we may take $D$ to be the negative of the exceptional divisor. 
map can be written as $F_{*}^{e}\left(J_{e} \omega_{W}\right)$, where $J_{e}=\pi_{*} \widetilde{O}_{\widetilde{W}}\left(K_{\widetilde{W} / W}+\left\lceil p^{e} G\right\rceil\right)$, and we deduce from the commutativity of (11) that

$$
\mathscr{g}\left(\mathfrak{b}^{\mu}\right) \subseteq J_{e}^{\left[1 / p^{e}\right]} .
$$

By Lemma 4.6 below, there is $r$ such that $\mathscr{g}\left(\mathfrak{b}^{m}\right) \subseteq \mathfrak{b}^{m-r}$ for every $m \geq r$. Since $-D$ is effective, by letting $e \gg 0$, we get

$$
J_{e}=\pi_{*} \mathscr{O}_{\widetilde{W}}\left(K_{\widetilde{W} / W}-\left\lfloor p^{e} \mu(Z-D)\right\rfloor\right) \subseteq \mathscr{g}\left(\mathfrak{b}^{\mu p^{e}}\right) \subseteq \mathfrak{b}^{\left\lfloor\mu p^{e}\right\rfloor-r},
$$

and therefore

$$
\mathscr{g}\left(\mathfrak{b}^{\lambda}\right)=\mathscr{g}\left(\mathfrak{b}^{\mu}\right) \subseteq\left(\mathfrak{b}^{\left\lfloor\mu p^{e}\right\rfloor-r}\right)^{\left[1 / p^{e}\right]} \subseteq \tau\left(\mathfrak{b}^{\frac{\left\lfloor\mu p^{e}\right\rfloor-r}{p^{e}}}\right) \subseteq \tau\left(\mathfrak{b}^{\lambda}\right),
$$

since $\lim _{e \rightarrow \infty}\left(\left\lfloor\mu p^{e}\right\rfloor-r\right) / p^{e}=\mu>\lambda$. This completes the proof.

Lemma 4.6. With the above notation, there is $r$ such that $\mathscr{f}\left(\mathfrak{b}^{m}\right) \subseteq \mathfrak{b}^{m-r}$ for every integer $m \geq r$.

Proof. It is enough to prove, more generally, that for every coherent sheaf $\mathscr{F}$ on $\widetilde{W}$, the graded module $M:=\oplus_{m \geq 0} \Gamma(\widetilde{W}, \mathscr{F}(-m Z))$ is finitely generated over the Rees algebra $S:=\oplus_{m \geq 0} \mathfrak{b}^{m}$. We may factor $\pi$ as

$$
\widetilde{W} \stackrel{g}{\rightarrow} B \stackrel{f}{\rightarrow} W,
$$

where $B$ is the normalized blow-up of $W$ along $\mathfrak{b}$ (that is, $B=\operatorname{Proj}\left(S^{\prime}\right)$, where $S^{\prime}$ is the normalization of $S$ ). The line bundle $\mathfrak{b} \cdot \mathscr{O}_{B}=O_{B}(-T)$ is ample over $W$, and using the projection formula we see that $M=\oplus_{m \geq 0} \Gamma\left(B, \pi_{*}(\mathscr{F}) \otimes \mathscr{O}_{B}(-m T)\right)$ is finitely generated over $S^{\prime}=\oplus_{m \geq 0} \Gamma\left(B, O_{B}(-m T)\right)$. Since $S^{\prime}$ is a finite $S$-algebra, it follows that $M$ is a finitely generated $S$-module.

We recall, for completeness, the proof of Proposition 4.4, which makes use of the de Rham complex $\Omega_{\widetilde{W}}^{\bullet}(\log (E))$ with log poles along the simple normal crossings divisor $E$. Note that while this complex does not have $0_{\widetilde{W}}$-linear differentials, its Frobenius push-forward $F_{*} \Omega_{\widetilde{W}}^{\bullet}(\log (E))$ does have this property. In particular, we may tensor this complex with line bundles. If $\mathscr{L}$ is a line bundle, then by the projection formula we have

$$
\left(F_{*} \Omega_{\widetilde{W}}^{i}(\log E)\right) \otimes \mathscr{L} \simeq F_{*}\left(\Omega_{\widetilde{W}}^{i}(\log E) \otimes \mathscr{L}^{p}\right) .
$$

The following facts are the key ingredients in the proof of Proposition 4.4.

(1) The Cartier isomorphism: There is a canonical isomorphism (see [Deligne and Illusie 1987, Theorem 1.2])

$$
C^{-1}: \Omega_{\widetilde{W}}^{i}(\log E) \simeq \mathscr{H}^{i} F_{*}\left(\Omega_{\widetilde{W}}^{\bullet}(\log E)\right) .
$$


(2) Insensitivity to small effective twists: Suppose that $B$ is an effective divisor supported on $E$, with all coefficients less than $p$. We have a twisted de Rham complex with $\log$ poles $\Omega_{\widetilde{W}}^{\bullet}(\log E)(B)$ (it is enough to check that the differential of the de Rham complex of meromorphic differential forms on $X$ preserves these subsheaves). In this case, the natural inclusion

$$
\Omega_{\widetilde{W}}^{\bullet}(\log E) \hookrightarrow \Omega_{\widetilde{W}}^{\bullet}(\log E)(B)
$$

is a quasiisomorphism; see [Hara 1998, Lemma 3.3; Mehta and Srinivas 1997, Corollary 4.2] for a proof. Combining this with the Cartier isomorphism, we find

$$
\Omega_{\widetilde{W}}^{i}(\log E) \simeq \mathscr{H}^{i}\left(F_{*}\left(\Omega_{\widetilde{W}}^{\bullet}(\log E)(B)\right)\right) .
$$

Proof of Proposition 4.4. Note first that it is enough to prove the case $e=1$. Indeed, if $\alpha_{G, e}$ is the morphism (10), we see that $\alpha_{G, e}=\alpha_{G, 1} \circ \alpha_{p G, 1} \circ \cdots \circ \alpha_{p^{e-1} G, 1}$, and the hypothesis implies that we may apply the condition for $e=1$ to each of $G, p G, \ldots, p^{e-1} G$. Therefore from now on we assume that $e=1$ (and in this case we will only need condition (A) for $\ell=1$ and condition (B) for $\ell=0$ ).

Let

$$
B:=(p-1) E+\lceil p G\rceil-p\lceil G\rceil=(p-1) E+p\lfloor-G\rfloor-\lfloor-p G\rfloor .
$$

Since $-G$ is effective, it follows from the second expression that $B$ is effective, and its coefficients are less than $p$. Let $K^{\bullet}:=F_{*} \Omega_{\widetilde{W}}^{\bullet}(\log E)(-E+\lceil p G\rceil)$. By tensoring (12) with $\widehat{O}_{\widetilde{W}}(-E+\lceil G\rceil)$, and using the projection formula, we get

$$
\Omega_{\widetilde{W}}^{i}(\log E)(-E+\lceil G\rceil) \simeq \mathscr{H}^{i}\left(F_{*}\left(\Omega_{\widetilde{W}}^{\bullet}(\log E)(B-p E+p\lceil G\rceil)\right)\right)=\mathscr{H}^{i}\left(K^{\bullet}\right) .
$$

Note that the morphism $\alpha_{G, 1}$ is identified to $\Gamma\left(\widetilde{W}, K^{n}\right) \rightarrow \Gamma\left(\widetilde{W}, \mathscr{H}^{n}\left(K^{\bullet}\right)\right)$. It is then straightforward to show, by breaking $K^{\bullet}$ into short exact sequences, and using the corresponding long exact sequences for cohomology, that $\alpha_{G, 1}$ is surjective if $H^{i}\left(\widetilde{W}, K^{n-i}\right)=0$ and $H^{i+1}\left(\widetilde{W}, \mathscr{H}^{n-i}\left(K^{\bullet}\right)\right)=0$ for all $i \geq 1$. By what we have seen, these are precisely conditions (A) with $\ell=1$ and (B) with $\ell=0$.

We will also make use of the following version of the Kodaira-Akizuki-Nakano vanishing theorem (in characteristic zero).

Theorem 4.7. Let $Y$ be a smooth, irreducible variety over a field $k$ of characteristic zero. If $Y$ is projective over an affine scheme $X, E$ is a reduced simple normal crossings divisor on $Y$, and $G$ is a $\mathbb{Q}$-divisor on $Y$ such that $G-\lfloor G\rfloor$ is supported on $E$ and $G$ is ample over $X$, then

$$
H^{i}\left(Y, \Omega_{Y}^{j}(\log E)(-E+\lceil G\rceil)\right)=0 \quad \text { if } i+j>\operatorname{dim}(X) .
$$


Proof. This is proved when $\operatorname{char}(k)=p>0$ in [Hara 1998, Corollary 3.8] under the assumption that $p>\operatorname{dim}(X)$ and that both $Y$ and $E$ admit a lifting to the second ring of Witt vectors $W_{2}(k)$ of $k$. The proof relies on an application of the results from [Deligne and Illusie 1987]. It is then standard to deduce the assertion in characteristic zero; see, for example, the proof of [ibid., Corollary 2.7].

We can now give the proof of our main result.

Proof of Theorem 4.1. Let $p_{m}=\operatorname{char}\left(L_{m}\right)$. We have by hypothesis $\lim _{m \rightarrow \infty} p_{m}=\infty$, hence $\operatorname{char}(k)=0$. In particular, there is a $\log$ resolution $\pi: Y \rightarrow X=\mathbb{A}_{k}^{n}$ of $\mathfrak{a}$. We write $\mathfrak{a} \cdot \mathrm{O}_{Y}=\mathrm{O}_{Y}(-Z)$, and let $E$ be a simple normal crossings divisor on $Y$ such that both $Z$ and $K_{Y / X}$ are supported on $E$. Let $\left[\pi_{m}\right]:\left[Y_{m}\right] \rightarrow\left[X_{m}\right]=\left[\mathbb{A}_{L_{m}}^{n}\right]$ be the corresponding morphism of internal schemes. It follows from Proposition 3.5 (iii) that if $Z_{\text {int }}=\left[Z_{m}\right]$, then $\mathfrak{a}_{m} \cdot \mathcal{O}_{Y_{m}}=\mathcal{O}\left(-Z_{m}\right)$ for almost all $m$. On the other hand, it is easy to deduce from Proposition 3.7 that $\left(K_{Y / X}\right)_{\text {int }}=\left[K_{Y_{m} / X_{m}}\right]$. If $E_{\text {int }}=\left[E_{m}\right]$, then $E_{m}$ has simple normal crossings for almost all $m$ by Proposition 3.9, and we conclude that $\pi_{m}$ is a $\log$ resolution of $\mathfrak{a}_{m}$ for almost all $m$. Moreover, if we use $\pi_{m}$ to define $\mathscr{E}\left(\mathfrak{a}_{m}^{\lambda}\right)$ on $X_{m}$, then we have $\mathscr{E}\left(\mathfrak{a}^{\lambda}\right)_{\text {int }}=\left[\mathscr{\mathscr { S }}\left(\mathfrak{a}_{m}^{\lambda}\right)\right]$ by Proposition 3.5 (iv).

For every $m$ such that $\pi_{m}$ gives a $\log$ resolution of $\mathfrak{a}_{m}$ we have $\tau\left(\mathfrak{a}_{m}^{\lambda}\right) \subseteq \mathscr{(}\left(\mathfrak{a}_{m}^{\lambda}\right)$ by Proposition 4.3. We now choose a rational number $\mu>\lambda$ such that $\mathscr{F}\left(\mathfrak{a}^{\lambda}\right)=\mathscr{F}\left(\mathfrak{a}^{\mu}\right)$, so that $\mathscr{f}\left(\mathfrak{a}_{m}^{\lambda}\right)=\mathscr{F}\left(\mathfrak{a}_{m}^{\mu}\right)$ for almost all $m$. We also choose a $\mathbb{Q}$-divisor $D$ supported on $E$ such that $-D$ is effective, $D$ is ample over $X$, and $\lceil\mu(D-Z)\rceil=\lceil-\mu Z\rceil$. We write $G=\mu(D-Z)$, and denote by $D_{m}$ and respectively $G_{m}$ the corresponding divisors on $Y_{m}$. It is clear that for almost all $m$ the divisor $-D_{m}$ is effective, $D_{m}$ is ample over $X_{m}$ (see Proposition $3.3(\mathrm{v})$ ), and $\left\lceil G_{m}\right\rceil=\left\lceil-\mu Z_{m}\right\rceil$. We deduce from Propositions 4.4 and 4.5 that $\mathscr{}\left(\mathfrak{a}_{m}^{\lambda}\right) \subseteq \tau\left(\mathfrak{a}_{m}^{\lambda}\right)$ if the following conditions hold:
$\left(\mathrm{A}_{m}\right) \quad H^{i}\left(Y_{m}, \Omega_{Y_{m}}^{n-i}\left(\log E_{m}\right)\left(-E_{m}+\left\lceil p_{m}^{\ell} G_{m}\right\rceil\right)\right)=0$ for all $i \geq 1$ and $\ell \geq 1$.
$\left(\mathrm{B}_{m}\right) \quad H^{i+1}\left(Y_{m}, \Omega_{Y_{m}}^{n-i}\left(\log E_{m}\right)\left(-E_{m}+\left\lceil p_{m}^{\ell} G_{m}\right\rceil\right)\right)=0$ for all $i \geq 1$ and $\ell \geq 0$.

It follows that in order to complete the proof, it is enough to show that conditions $\left(\mathrm{A}_{m}\right)$ and $\left(\mathrm{B}_{m}\right)$ hold for almost all $m$.

Note first that by Theorem 4.7, we have $H^{i+1}\left(Y, \Omega_{Y}^{n-i}(\log E)(-E+\lceil G\rceil)\right)=0$ for all $i \geq 0$. Using Proposition 3.5 (iv), we deduce that

$$
H^{i+1}\left(Y_{m}, \Omega_{Y_{m}}^{n-i}\left(\log E_{m}\right)\left(-E_{m}+\left\lceil G_{m}\right\rceil\right)\right)=0
$$

for all $i \geq 0$ and almost all $m$ (since these groups vanish automatically when $i \geq n$, we only need to consider finitely many such $i$ ). This takes care of the condition $\left(\mathrm{B}_{m}\right)$ for $\ell=0$.

We now treat the remaining conditions. Let us fix a positive integer $d$ such that $d G$ is an integral divisor. Let $\mathscr{F}_{1}, \ldots, \mathscr{F}_{M}$ denote the sheaves $\Omega_{Y}^{t}(\log E)(-E+\lceil s G\rceil)$, for integers $0 \leq s \leq d-1$ and $0 \leq t \leq n$. Since $d G$ is ample over the affine variety 
$X$, there is $j_{0}$ such that $H^{i}\left(Y, \mathscr{F}_{t}(j d G)\right)=0$ for every $j \geq j_{0}$, every $i \geq 1$ and every $t \leq M$. If $m$ is such that $p_{m} \geq\left(j_{0}+1\right) d$, and if for $\ell \geq 1$ we take $s$ with $0 \leq s \leq d-1$ such that $p_{m}^{\ell} \equiv s(\bmod d)$, then

$$
\left\lceil p_{m}^{\ell} G\right\rceil=\frac{p_{m}^{\ell}-s}{d}(d G)+\lceil s G\rceil \text { and } \quad \frac{p_{m}^{\ell}-s}{d} \geq \frac{p_{m}-s}{d} \geq j_{0} .
$$

We deduce from Corollary 3.6 that the vanishings in $\left(\mathrm{A}_{m}\right)$ and $\left(\mathrm{B}_{m}\right)$ hold when $\ell \geq 1$ for almost all $m$ (note that for such $m$ we may assume that $p_{m} \geq\left(j_{0}+1\right) d$ ). This completes the proof of the theorem.

\section{Acknowledgments}

Our project started during the "Mathematics Research Community" on Commutative Algebra in Snowbird, Utah, 2010. We are grateful to the AMS for setting up this program, and for providing a stimulating research environment.

\section{References}

[Blickle et al. 2008] M. Blickle, M. Mustață, and K. E. Smith, "Discreteness and rationality of F-thresholds", Michigan Math. J. 57 (2008), 43-61. MR 2010c:13003 Zbl 1177.13013

[Blickle et al. 2009] M. Blickle, M. Mustață, and K. E. Smith, " $F$-thresholds of hypersurfaces”, Trans. Amer. Math. Soc. 361:12 (2009), 6549-6565. MR 2011a:13006 Zbl 1193.13003

[Blickle et al. 2010] M. Blickle, K. Schwede, S. Takagi, and W. Zhang, "Discreteness and rationality of $F$-jumping numbers on singular varieties", Math. Ann. 347:4 (2010), 917-949. MR 2011k:13008 Zbl 1198.13007

[Deligne and Illusie 1987] P. Deligne and L. Illusie, "Relèvements modulo $p^{2}$ et décomposition du complexe de de Rham”, Invent. Math. 89:2 (1987), 247-270. MR 88j:14029 Zbl 0632.14017

[van den Dries and Schmidt 1984] L. van den Dries and K. Schmidt, "Bounds in the theory of polynomial rings over fields: A nonstandard approach”, Invent. Math. 76:1 (1984), 77-91. MR 85i:12016 Zbl 0539.13011

[Ein and Mustață 2006] L. Ein and M. Mustață, "Invariants of singularities of pairs”, pp. 583-602 in International Congress of Mathematicians (Madrid, 2006), vol. 2, edited by M. Sanz-Solé et al., European Mathematical Society, Zürich, 2006. MR 2007m:14050 Zbl 1096.14030

[de Fernex and Mustață 2009] T. de Fernex and M. Mustață, "Limits of log canonical thresholds", Ann. Sci. Éc. Norm. Supér. (4) 42:3 (2009), 491-515. MR 2010i:14030 Zbl 1186.14007

[Goldblatt 1998] R. Goldblatt, Lectures on the hyperreals: An introduction to nonstandard analysis, Graduate Texts in Mathematics 188, Springer, New York, 1998. MR 2000a:03113 Zbl 0911.03032

[Hara 1998] N. Hara, "A characterization of rational singularities in terms of injectivity of Frobenius maps”, Amer. J. Math. 120:5 (1998), 981-996. MR 99h:13005 Zbl 0942.13006

[Hara and Watanabe 2002] N. Hara and K.-I. Watanabe, "F-regular and F-pure rings vs. log terminal and log canonical singularities", J. Algebraic Geom. 11:2 (2002), 363-392. MR 2002k:13009 Zbl 1013.13004

[Hara and Yoshida 2003] N. Hara and K.-I. Yoshida, "A generalization of tight closure and multiplier ideals", Trans. Amer. Math. Soc. 355:8 (2003), 3143-3174. MR 2004i:13003 Zbl 1028.13003 
1482 Bhargav Bhatt, Daniel Hernández, Lance Edward Miller and Mircea Mustață

[Kollár 1997] J. Kollár, "Singularities of pairs", pp. 221-287 in Algebraic geometry, Part 1 (Santa Cruz, CA, 1995), edited by J. Kollár et al., Proc. Sympos. Pure Math. 62, Amer. Math. Soc., Providence, RI, 1997. MR 99m:14033 Zbl 0905.14002

[Lazarsfeld 2004] R. Lazarsfeld, Positivity in algebraic geometry, II: Positivity for vector bundles, and multiplier ideals, Ergeb. Math. Grenzgeb. (3) 49, Springer, Berlin, 2004. MR 2005k:14001b Zbl 1093.14500

[Mehta and Srinivas 1997] V. B. Mehta and V. Srinivas, "A characterization of rational singularities", Asian J. Math. 1:2 (1997), 249-271. MR 99e:13009 Zbl 0920.13020

[Mustață et al. 2005] M. Mustaţă, S. Takagi, and K.-i. Watanabe, "F-thresholds and Bernstein-Sato polynomials", pp. 341-364 in European Congress of Mathematics (Stockholm, 2004), edited by A. Laptev, European Mathematical Society, Zürich, 2005. MR 2007b:13010 Zbl 1092.32014

[Schoutens 2005] H. Schoutens, "Log-terminal singularities and vanishing theorems via non-standard tight closure", J. Algebraic Geom. 14:2 (2005), 357-390. MR 2006e:13005 Zbl 1070.14005

[Schoutens 2010] H. Schoutens, The use of ultraproducts in commutative algebra, Lecture Notes in Mathematics 1999, Springer, Berlin, 2010. MR 2012g:13046 Zbl 1205.13002

[Takagi and Watanabe 2004] S. Takagi and K.-i. Watanabe, "On F-pure thresholds", J. Algebra 282:1 (2004), 278-297. MR 2006a:13010 Zbl 1082.13004

Communicated by Craig Huneke

Received 2011-06-01 Revised 2011-11-16 Accepted 2011-12-20

bhargav.bhatt@gmail.com Department of Mathematics University of Michigan, Ann Arbor, MI 48109, United States

Current address:

School of Mathematics, Institute for Advanced Study, Princeton, NJ 08540, United States

dhernan@umn.edu

Department of Mathematics, University of Minnesota, Minneapolis, MN 55455, United States

Imiller@math.utah.edu

Department of Mathematics, University of Utah, Salt Lake City, UT 84112, United States

mmustata@umich.edu

Department of Mathematics, University of Michigan, Ann Arbor, MI 48109, United States 


\section{Algebra \& Number Theory}

msp.berkeley.edu/ant

\section{EDITORS}

MANAGING EDITOR

Bjorn Poonen

Massachusetts Institute of Technology

Cambridge, USA

\author{
EDITORIAL BOARD CHAIR \\ David Eisenbud \\ University of California \\ Berkeley, USA
}

\section{BOARD OF EDITORS}

Georgia Benkart

Dave Benson

Richard E. Borcherds

John H. Coates

J-L. Colliot-Thélène

Brian D. Conrad

Hélène Esnault

Hubert Flenner

Edward Frenkel

Andrew Granville

Joseph Gubeladze

Ehud Hrushovski

Craig Huneke

Mikhail Kapranov

Yujiro Kawamata

János Kollár

Yuri Manin

Barry Mazur

Philippe Michel
University of Wisconsin, Madison, USA

University of Aberdeen, Scotland

University of California, Berkeley, USA

University of Cambridge, UK

CNRS, Université Paris-Sud, France

University of Michigan, USA

Freie Universität Berlin, Germany

Ruhr-Universität, Germany

University of California, Berkeley, USA

Université de Montréal, Canada

San Francisco State University, USA

Hebrew University, Israel

University of Virginia, USA

Yale University, USA

University of Tokyo, Japan

Princeton University, USA

Northwestern University, USA

Harvard University, USA

École Polytechnique Fédérale de Lausanne
Susan Montgomery

Shigefumi Mori

Raman Parimala

Jonathan Pila

Victor Reiner

Karl Rubin

Peter Sarnak

Joseph H. Silverman

Michael Singer

Vasudevan Srinivas

J. Toby Stafford

Bernd Sturmfels

Richard Taylor

Ravi Vakil

Michel van den Bergh

Marie-France Vignéras

Kei-Ichi Watanabe

Andrei Zelevinsky

Efim Zelmanov
University of Southern California, USA

RIMS, Kyoto University, Japan

Emory University, USA

University of Oxford, UK

University of Minnesota, USA

University of California, Irvine, USA

Princeton University, USA

Brown University, USA

North Carolina State University, USA

Tata Inst. of Fund. Research, India

University of Michigan, USA

University of California, Berkeley, USA

Harvard University, USA

Stanford University, USA

Hasselt University, Belgium

Université Paris VII, France

Nihon University, Japan

Northeastern University, USA

University of California, San Diego, USA

\section{PRODUCTION}

production@msp.org

Silvio Levy, Scientific Editor

See inside back cover or www.jant.org for submission instructions.

The subscription price for 2012 is US \$175/year for the electronic version, and \$275/year ( $\$ 40$ shipping outside the US) for print and electronic. Subscriptions, requests for back issues from the last three years and changes of subscribers address should be sent to Mathematical Sciences Publishers, Department of Mathematics, University of California, Berkeley, CA 94720-3840, USA.

Algebra \& Number Theory (ISSN 1937-0652) at Mathematical Sciences Publishers, Department of Mathematics, University of California, Berkeley, CA 94720-3840 is published continuously online. Periodical rate postage paid at Berkeley, CA 94704, and additional mailing offices.

ANT peer review and production are managed by EditFLOW ${ }^{\circledR}$ from Mathematical Sciences Publishers.

PUBLISHED BY

mathematical sciences publishers

http://msp.org/

A NON-PROFIT CORPORATION

Typeset in IATEX

Copyright ( 2012 by Mathematical Sciences Publishers 


\section{Algebra \& Number Theory}

\section{Volume $6 \quad$ No. $7 \quad 2012$}

On the rank of the fibers of rational elliptic surfaces

Cecília Salgado

Néron's pairing and relative algebraic equivalence

CÉDRIC PÉPIN

Free subalgebras of quotient rings of Ore extensions

JASON P. BELL and DANIEL ROGALSKI

Classes de cycles motiviques étales

1369

BRUNO KAHN

Higher-order Maass forms

1409

ROELOF BRUGGEMAN and NiKOLAOS DIAMANTIS

Log canonical thresholds, $F$-pure thresholds, and nonstandard extensions

Bhargav Bhatt, Daniel J. Hernández, Lance Edward Miller and Mircea MUSTAȚĂ

The semistable reduction problem for the space of morphisms on $\mathbb{P}^{n}$

ALON LEVY

Grothendieck's trace map for arithmetic surfaces via residues and higher adèles

MATTHEW MORROW

Crystalline extensions and the weight part of Serre's conjecture

Toby GeE, Tong LiU and DAVID SAVITT

Annihilating the cohomology of group schemes 